\title{
Convectively induced shear instability in large amplitude internal solitary waves
}

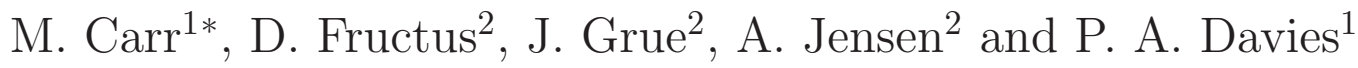 \\ ${ }^{1}$ Department of Civil Engineering, University of Dundee, \\ Dundee, DD1 4HN, U.K. \\ ${ }^{2}$ Mechanics Division, Department of Mathematics, \\ University of Oslo, Oslo, Norway. \\ * Corresponding author, now at \\ School of Mathematics \& Statistics, University of St Andrews, \\ St Andrews, KY16 9SS, U.K. \\ email: magda@mcs.st-and.ac.uk, \\ Phone:+44(1334)463715, Fax:+44(1334)463748
}

October 20, 2008

\begin{abstract}
Laboratory study has been carried out to investigate the instability of an internal solitary wave of depression in a shallow stratified fluid system. The experimental campaign has been supported by theoretical computations and has focused on a two layered stratification consisting of a homogeneous dense layer below a linearly stratified top layer. The initial background stratification has been varied and it is found that the onset, and intensity of breaking are affected dramatically by changes in the background stratification. Manifestations of a combination of shear and convective instability are seen on the leading face of the wave. It is shown that there is interplay between the two instability types and convective instability induces shear by enhancing isopycnal
\end{abstract}


compression. Variation of the upper boundary condition is also found to have an effect on stability. In particular, the implications for convective instability are shown to be profound and a dramatic increase in wave amplitude is seen for a fixed (as opposed to free) upper boundary condition.

Keywords: Internal Solitary Wave, Convective Instability, Shear Instability.

\section{INTRODUCTION}

Internal solitary waves (ISWs) are ubiquitous features in the Earth's atmosphere and ocean (for example, see the recent review by Helfrich \& Melville ${ }^{1}$ ). Breaking ISWs can result in significant vertical mixing in the environment in which they propagate. They are an important source of mixing, turbulence, and mass and momentum transfer. In physical oceanography, one of the most topical issues of debate is the role of unstable ISWs in the overall mixing of coastal oceans - a process that, in turn, has implications for global ocean circulation and climate modelling. To understand the behavior of ISWs in this context, it is imperative that the evolutionary processes that lead to breaking and the subsequent generation of turbulence are better understood. Despite acknowledgment of this in the literature, relatively little is known about the instability of large amplitude ISWs in shallow fluid systems.

Due to the difference in scale at which breaking is thought to take place and the scale at which large amplitude ISWs have been sampled, very limited field data are available for reference. The cleanest observation of a breaking ISW of depression is that of Moum et al. ${ }^{2}$ Figure 14 of their work shows a beautiful acoustical backscatter image of what appears to be a manifestation of a shear-induced (Kelvin-Helmholtz) instability in an ISW propagating

shoreward over the Oregon continental shelf. The instability captured by Moum et al. ${ }^{2}$ 
was associated with compressive wave straining deduced from measurements of isopycnal compression. In a recent discussion Farmer (private communication) speculated that the instability may be due to the presence of pools of fresh water in the surface region from a nearby river. In an effort to understand better such breaking in particular and instability in general, a laboratory campaign supported by theoretical development is presented.

The undisturbed background stratification in Moum et al. ${ }^{2}$ can be approximated by a stably stratified two layer fluid consisting of a homogeneous dense layer below a linearlystratified top layer (see Fig. 8 of their work). Such a density configuration has been reported in other works where ISWs are observed frequently in nature (see Apel et al.; ${ }^{3}$ Farmer \& Smith $;{ }^{4}$ Grue et al., ${ }^{5}$ for example). Grue et al. ${ }^{5}$ also considered the propagation of an ISW in a two layer configuration. In waves of large amplitude they observed trapped cores in which convective breaking took place through the formation of small vortices in the leading part of the wave. Such instability is caused by horizontal advection of density and for an ISW occurs when the horizontal particle velocity exceeds the wave velocity. This instability is referred to as convective instability. In non-breaking waves individual streamlines, are smooth and nonvertical. Grue et al. ${ }^{5}$ observed closed streamlines in their breaking cases, resulting in local overturning associated with local peaks in the vorticity. Similar behavior has been observed in computations of large solitary waves that break (see Lamb; ${ }^{6}$ Lamb \& Wilkie; ${ }^{7}$ Fructus \& Grue $\left.^{8}\right)$.

In the laboratory observations presented here, a different dynamic is seen. A combination of convective (local overturning) and shear instability is found. This is the first time evidence of such instability has been seen and in particular the first time shear instability has been observed in the leading half of the wave. The data show that there is interaction between the shear and convective instabilities observed. The breaking observed here is fundamentally different from that reported in Grue et al. ${ }^{5}$

In the present study, most experiments have been performed with a fixed upper boundary, 
to enable comparisons to be made with predictions from numerical and oceanic models incorporating rigid lid conditions. However, comparison is also made here with some free surface counterpart cases and it is shown that the breaking dynamics are significantly different in the two regimes. Grue et al. ${ }^{5}$ found that convective instability in moderate amplitude waves, with wave-induced velocity less than the wave speed, disappeared if the upper boundary was fixed as opposed to free. This led the authors to speculate that the convective instability they saw (their fig 13) was associated with surface tension effects. There is some discrepancy between the experimental results of Grue et al. ${ }^{5}$ with a free surface and the fully nonlinear theoretical predictions of Fructus \& Grue. ${ }^{8}$ Specifically, the critical wave amplitude suggested by the theory underpredicts that inferred from the experimental observations. It is shown here that the rigid lid approximation is responsible for the discrepancy; in particular the study demonstrates for the first time that convective instability and wave amplitude are directly affected by the upper boundary condition. This finding is of significance to mathematical modelers concerned with the analysis of ISWs.

The present paper focuses on a two layer regime; the instability of ISWs in a three layer configuration is addressed separately in Fructus et al. ${ }^{9}$ who consider a stratification consisting of a linearly stratified middle layer sandwiched between homogeneous top and bottom layers. For stable (i.e. non-breaking) waves, Fructus et al. ${ }^{9}$ found excellent agreement between fully nonlinear theory and experimental data enabling the numerical model to be exploited to predict, for each experimental wave, the value of the Richardson number throughout the wave domain. Unambiguously associating the onset of shear instability (and breaking) with a fully resolved local value of the Richardson number led to significant new insight in the three layer regime. A similar theoretical approach is taken here. The difference in stability characteristics between the two and three layer regimes is discussed in section III A and some three layer experiments are presented in section III B to aid interpretation of the two layer counterpart flows and the effect upon such cases of the form of the upper 
(a)

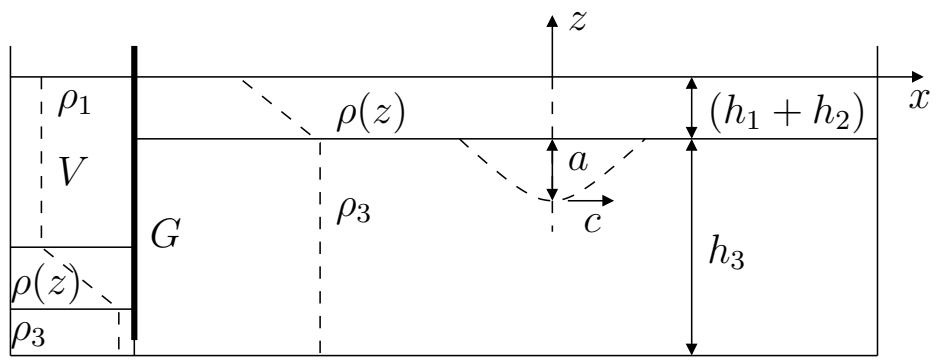

(b)

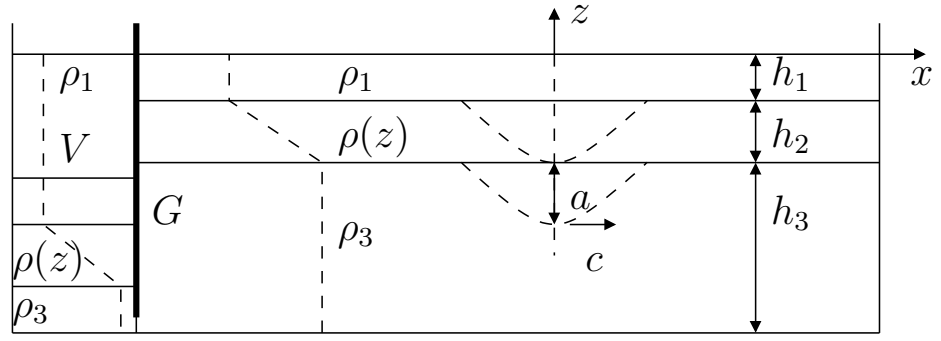

Figure 1: Schematic diagram of the experimental set up (a) 2 layer configuration (b) 3 layer configuration.

boundary condition.

The paper is laid out as follows. In the next section the laboratory facilities and measurement techniques are described. In section III a description of the experimental observations is given and in section IV the observations are explained in light of theoretical development. Comparison with the field observations of Moum et al. ${ }^{2}$ is given in section $\mathrm{V}$, and finally some conclusions are drawn in section VI.

\section{THE EXPERIMENTAL STUDY}

\section{A. Model configuration and experimental arrangement}

Figure 1 shows a sketch of the experimental arrangements for both two and three layer configurations, together with a definition of the $(x, z)$ coordinate system of the reference frame. Within a Cartesian coordinate system $(x, y, z)$, the $x$ and $z$ directions denote respectively the (horizontal) direction of propagation of the wave and the direction anti-parallel to the 
gravitational acceleration vector $\mathbf{g}=(0,0,-g)$. The origin is chosen such that $x=0$ represents the horizontal position of the wave trough and $z=0$ the upper boundary of the water column. In the two layer regime the background stratification consists of a miscible homogeneous lower layer of fluid of depth $h_{3}$ and density $\rho_{3}$ superposed by a linearly stratified top layer of depth $\left(h_{1}+h_{2}\right)$ in which the density $\rho(z)$ is a linear function of $z$. In the three layer regime the background stratification consists of upper and lower layers of miscible homogeneous fluid of density $\rho_{1}$ and $\rho_{3}$ respectively and undisturbed thickness $h_{1}$ and $h_{3}$ respectively. The pycnocline has an undisturbed thickness of $h_{2}$ and the density, $\rho(z)$, varies as a linear function of $z$. An ISW of amplitude $a$ is generated on the pycnocline and it travels along the interface with celerity $c$. The flow is two-dimensional, with no variation in the cross-flow $(y)$ direction.

The experiments were conducted using two wave tanks of dimensions $12.6 \mathrm{~m} \times 0.5 \mathrm{~m} \times 1.0 \mathrm{~m}$ and $6.4 \mathrm{~m} \times 0.4 \mathrm{~m} \times 0.6 \mathrm{~m}$ (length, width and depth, respectively). Experiments were performed in the big tank to check the fundamental stability characteristics and resolution of the small tank. Excellent agreement between the two was found. Only results from the small tank are presented here. Data from the large tank in the three layer configuration can be found in Fructus et al. ${ }^{9}$ The lower layer was filled first with a prepared solution of brine of prescribed density $\rho_{3}$. The top layer was then carefully added via a floating sponge arrangement (see Grue et al. ${ }^{5}$ ). The double bucket technique was used to obtain the linearly-stratified layer, see for example Fortuin. ${ }^{10}$ The form of the stratification was pre-set for a given experiment by careful adjustment of the initial volume and densities used in fresh and saline water supply reservoirs. In the small tank, the profile of the stratification was measured via an array of high precision micro-conductivity probes, see Davies. ${ }^{11}$ In the large tank, Yokogawa SC12 and Mettler-Toldeo DA-300M meters were used.

Waves of very large amplitude were generated using the so called step pool technique. After the layers had been filled, a gate $G$ was inserted at one end of the tank, with a gap of 
approximately $5 \mathrm{~mm}$ being left between the bed of the tank and the bottom of the gate. A fixed volume $V$ of brine with density $\rho_{1}$ (where $\rho_{1}$ is the density at the surface) was then added behind the gate (see Fig. 1). Upon release of the gate, a single solitary wave of depression of very large amplitude was quickly generated. The leading part of the wave almost instantly attained the shape of a wave in steady motion (see also Grue et al. ${ }^{5,12}$ ). Focus was on generating ISWs of very large amplitude. This was achieved by careful adjustment of the initial volume $V$ and, in particular, its width to depth ratio, such that the vast part of the initial potential energy behind the gate went into the ISW. In the case of an initially long, shallow volume, the KdV scenario occurs, whereby a sequence of weakly nonlinear waves are generated (see Kao et al. ${ }^{13}$ ). A total of 96 experiments were conducted; 31 in the two layer regime and 65 in the three layer regime, from which 35 have been selected for presentation and discussion here.

\section{B. Measurement technique}

Particle image velocimetry (PIV) was used to visualize and quantify a given two-dimensional $(x, z)$ slice of the flow field $(u, w)$. To implement PIV, a vertical section in the mid-plane of the tank (where edge wall effects are assumed negligible) was illuminated. In the small tank this was achieved by a continuous, collimated light sheet from an array of light boxes placed below the (transparent) base of the tank. The light sheet had a thickness of approximately $10 \mathrm{~mm}$ and it illuminated a section of the tank $1.4 \mathrm{~m}$ long and $0.6 \mathrm{~m}$ deep. In the large tank a $100 \mathrm{~Hz}$ Nd:YAG, 15 mJ per pulse laser illuminated a section approximately $0.5 \mathrm{~m}$ long, $2 \mathrm{~mm}$ thick and $1.0 \mathrm{~m}$ deep. The illuminated sections were seeded with neutrally-buoyant, light-reflecting tracer particles of "Pliolite" having diameters in the range $150-300 \mu \mathrm{m}$ in the small tank and $500-700 \mu \mathrm{m}$ in the large tank. Motions within the vertical light sheet were viewed and recorded from the side using a fixed digital video camera set up outside the tank. The camera had a spatial resolution and capture rate of $1372 \times 1372$ pixels and 
24 frames per second respectively, in the small tank and $1024 \times 1024$ pixels and 99 frames per second respectively, in the large tank. The dynamics of interest occurred mainly in the top layer. The camera was positioned level with the surface of the undisturbed flow to avoid distortion and perspective errors in this portion of the flow field. The resulting video record of the flow within the illuminated window was processed using the software package DigiFlow (see Dalziel ${ }^{14}$ ) to generate continuous synoptic velocity field data throughout the water column. In all cases, the recording system was stationary with respect to the tank and the ISW traveled through the illuminated measurement window. The horizontal location of the measurement window was carefully chosen such that (i) the wave was fully developed by the time it was observed and (ii) the end wall of the tank did not interfer with the dynamics under consideration. The viewing location was chosen to be $2 / 3$ downstream of the end of the sorting distance and $1 / 3$ upstream of the end wall of the tank.

The development of the interface was monitored using the time series function of DigiFlow, by tracking the changes with time of the pixel values in a given column of the digitized image. The image was probed using DigiFlow and from it an estimate of the amplitude and the time at which the interface reached maximum displacement were made. This process was repeated at three fixed locations $x_{1,2,3}$ over a known horizontal distance $\Delta x$ of approximately $2 h_{3}$ in the small tank and $h_{3} / 2$ in the large tank $\left(h_{3}\right.$ was typically $30 \mathrm{~cm}$ in the small tank and $64 \mathrm{~cm}$ in the large tank). This yielded an average amplitude $a_{\text {exp }}$ and estimate of the celerity $c_{\text {exp }}(=\Delta x / \Delta t)$ of the wave by noting the average time $\Delta t$ between maximal interface displacement at the three fixed locations $x_{1,2,3}$. Small decay in amplitude and wave speed between the three measurement locations was seen as the wave propagated along the tank. In the two layer regime, variation produced an error of approximately $1.9 \%$ in measuring $a_{\text {exp }}$ and $1.5 \%$ in measuring $c_{\text {exp }}$. The difference in stratification, between the bottom and top of the water column, gave a maximum error of $1 \%$ in PIV measurments of displacement due to variation in refractive index. Hence the PIV velocity field measurements are accurate 
to within $1 \%$.

\section{EXPERIMENTAL OBSERVATIONS}

\section{A. Instability in the 2 layer regime - fixed upper boundary condition}

Table I. The instability of an ISW in a 2 layer configuration consisting of a homogeneous bottom layer and a linearly stratified top layer with a fixed upper boundary condition.

\begin{tabular}{|c|c|c|c|c|c|c|c|c|}
\hline Date & $\begin{array}{l}h_{3} \\
(\mathrm{~m})\end{array}$ & $\begin{array}{c}h_{1}+h_{2} \\
(\mathrm{~m})\end{array}$ & $h_{3}^{*}$ & $a_{e x p}^{*}$ & $\begin{array}{c}c_{e x p} \\
\left(\mathrm{~ms}^{-1}\right)\end{array}$ & $c_{\exp } / c_{0}$ & $\begin{array}{c}N \\
\left(\mathrm{~s}^{-1}\right)\end{array}$ & Instability Observed \\
\hline $01 / 06 / 05$ & 0.293 & 0.077 & 3.81 & $1.63 \pm 0.03$ & $0.115 \pm 0.002$ & $1.74 \pm 0.03$ & 1.48 & Moderate \\
\hline $24 / 04 / 06$ & 0.290 & 0.075 & 3.87 & $1.59 \pm 0.03$ & $0.111 \pm 0.002$ & $1.66 \pm 0.03$ & 1.50 & Moderate \\
\hline $15 / 06 / 05$ & 0.290 & 0.075 & 3.87 & $1.45 \pm 0.03$ & $0.108 \pm 0.002$ & $1.53 \pm 0.02$ & 1.53 & Slight-Moderate \\
\hline $25 / 04 / 06$ & 0.287 & 0.080 & 3.59 & $1.23 \pm 0.02$ & $0.098 \pm 0.002$ & $1.50 \pm 0.02$ & 1.45 & Slight-Moderate \\
\hline $11 / 08 / 05$ & 0.286 & 0.077 & 3.71 & $1.07 \pm 0.02$ & $0.104 \pm 0.002$ & $1.49 \pm 0.02$ & 1.50 & Very Slight \\
\hline $14 / 06 / 05$ & 0.294 & 0.078 & 3.77 & $0.84 \pm 0.02$ & $0.095 \pm 0.001$ & $1.42 \pm 0.02$ & 1.55 & None \\
\hline $26 / 04 / 06$ & 0.294 & 0.052 & 5.65 & $2.44 \pm 0.05$ & $0.093 \pm 0.001$ & $1.92 \pm 0.03$ & 1.47 & Moderate-Vigorous \\
\hline $28 / 04 / 06$ & 0.290 & 0.055 & 5.27 & $1.97 \pm 0.04$ & $0.093 \pm 0.001$ & $1.93 \pm 0.03$ & 1.43 & Moderate-Vigorous \\
\hline $27 / 04 / 06$ & 0.292 & 0.054 & 5.41 & $1.16 \pm 0.02$ & $0.076 \pm 0.001$ & $1.62 \pm 0.02$ & 1.44 & Very Slight \\
\hline 01/05/06 & 0.289 & 0.038 & 7.61 & $3.17 \pm 0.06$ & $0.069 \pm 0.001$ & $2.22 \pm 0.03$ & 1.36 & Vigorous \\
\hline $03 / 05 / 06$ & 0.289 & 0.037 & 7.81 & $2.62 \pm 0.05$ & $0.064 \pm 0.001$ & $2.01 \pm 0.03$ & 1.38 & Moderate-Vigorous \\
\hline $02 / 05 / 06$ & 0.290 & 0.036 & 8.06 & $1.95 \pm 0.04$ & $0.045 \pm 0.001$ & $1.42 \pm 0.02$ & 1.35 & Very Slight \\
\hline $16 / 06 / 05$ & 0.292 & 0.026 & 11.23 & $4.47 \pm 0.08$ & $0.095 \pm 0.001$ & $2.52 \pm 0.04$ & 2.34 & Vigorous \\
\hline $20 / 07 / 05$ & 0.292 & 0.025 & 11.68 & $3.04 \pm 0.06$ & $0.087 \pm 0.001$ & $2.25 \pm 0.03$ & 2.62 & Vigorous \\
\hline $23 / 06 / 05$ & 0.290 & 0.026 & 11.15 & $2.33 \pm 0.04$ & $0.080 \pm 0.001$ & $2.10 \pm 0.03$ & 2.51 & Slight-Moderate \\
\hline $12 / 08 / 05$ & 0.290 & 0.026 & 11.15 & $1.73 \pm 0.03$ & $0.076 \pm 0.001$ & $1.74 \pm 0.03$ & 2.65 & Very Slight \\
\hline
\end{tabular}

Table I is split into four data blocks and presents the experimentally-observed stability characteristics of an ISW propagating in the two layer configuration. The depth of the top layer is used as a length scale throughout and starred variables refer to quantities nondimensionalized by $h_{c}=\left(h_{1}+h_{2}\right)$. The upper boundary was held fixed by placing a Styrofoam lid on top of the free surface after filling the channel and before wave generation. In the first data block, $h_{3}^{*}, c_{\exp }$ and $N$ (the Brunt-Väisälä frequency in the upper layer) are approximately $3.77,0.11 \mathrm{~ms}^{-1}$ and $1.50 \mathrm{~s}^{-1}$ respectively, and the amplitude of the wave $a_{\text {exp }}^{*}$, is varied. In the large-moderate amplitude cases (first four experiments), instability appeared to be a combination of shear and convective. Mixing was confined to the top layer, and the lower interface was not disturbed by the short scale instabilities. The onset of instability was toward the front of the wave just below the upper boundary. Figures 2-7 illustrate the 
instability seen in experiment 24/04/06 at sequential time intervals as an example. Note the motion induced by convective instablility is not obvious from Figs. 2-7 since the motion occurs on a relatively small scale and is not clearly identifiable to the untrained eye. Motion induced by shear instability is easier to identify as it takes the form of billowing and is on a larger scale. Unless stated otherwise, the wave is propagating from left to right in all figures shown. The lower layer was recycled between experimental runs while the top layer was added fresh each time. As a result the lower layer was cloudier than the top layer and the interface can clearly be identified. The lighter shaded area running along the bottom of the frame was due to a change in material on the back wall of the tank and is not associated with the flow dynamics. In the upper layer a trapped core of unstable fluid being transported with the wave was identified by a further cloudy region, in which the tracer particles look slightly blurred as a result of small scale (convective) mixing. This unstable region began at $\left(x^{*}, z^{*}\right) \approx(4.5,-0.2)$ and extended throughout the top layer in the negative $x$ and $z$ directions away from this point. Instability began at this point as convective overturning (small scale mixing, a result of the local fluid velocity exceeding the wave speed) and quickly developed in a shear-billow-like fashion on the front face of the wave. Two such billows can be seen in Fig. 4 at $\left(x^{*}, z^{*}\right) \approx(3.3,-1.6)$ and $\left(x^{*}, z^{*}\right) \approx(1.6,-1.9)$ for example. The ensuing flow was a combination of both convective overturning and shear billowing. The manifestation of the combined instability extended in the negative $x$ direction and remained confined to the top layer, as illustrated at later times in Figs. 5-7. Note the distinctly stable region between the interface and the core of unstable fluid above. In addition, note that Figs. 2-5 show compression of the isopycnals in the front half of the wave. A schematic summary of the instability seen is given in Fig. 8.

It is conjectured that the shear-billow-like instability seen on the leading face of the wave is somewhat induced by the preceeding convective overturning in the top of the water column. Shear instability can be analyzed in terms of the Richardson number $R i=g \beta /(\partial u / \partial z)^{2}$ 


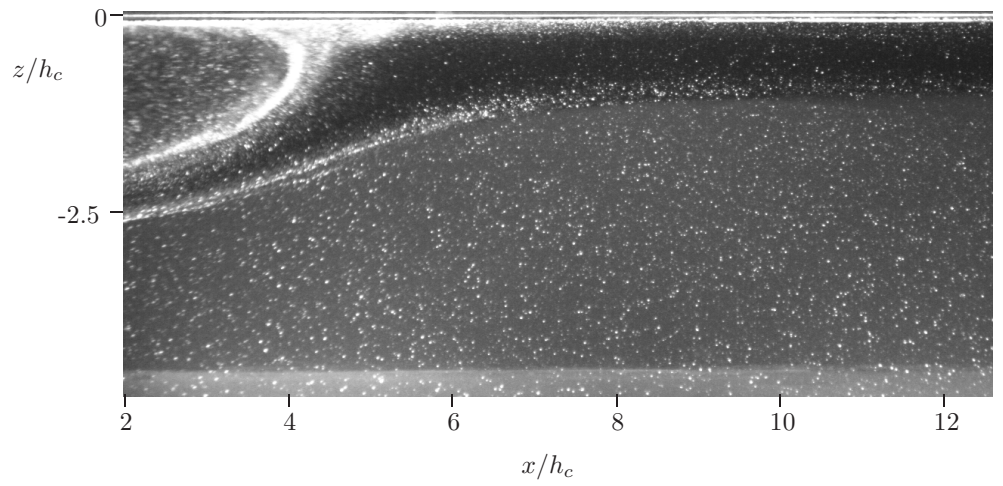

Figure 2: Frame 167 from experimental movie 24/04/06.

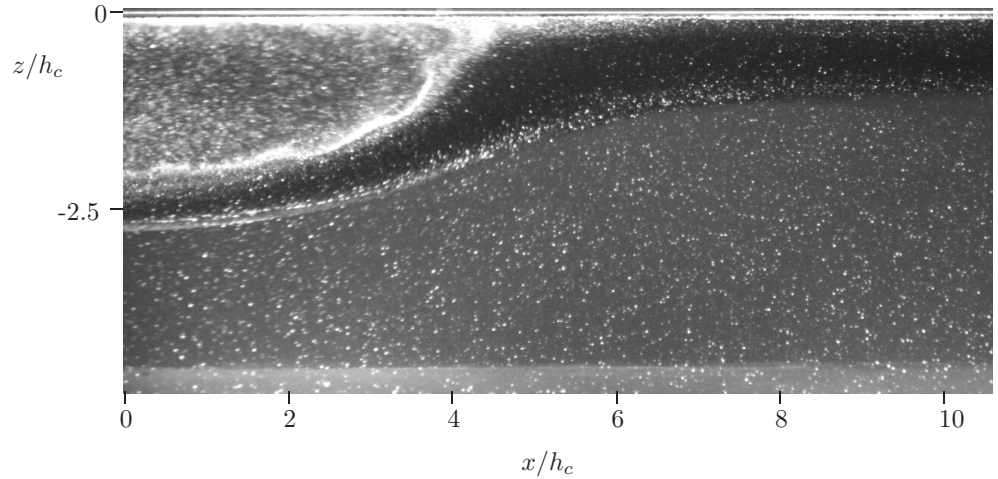

Figure 3: Frame 197 from experimental movie 24/04/06.

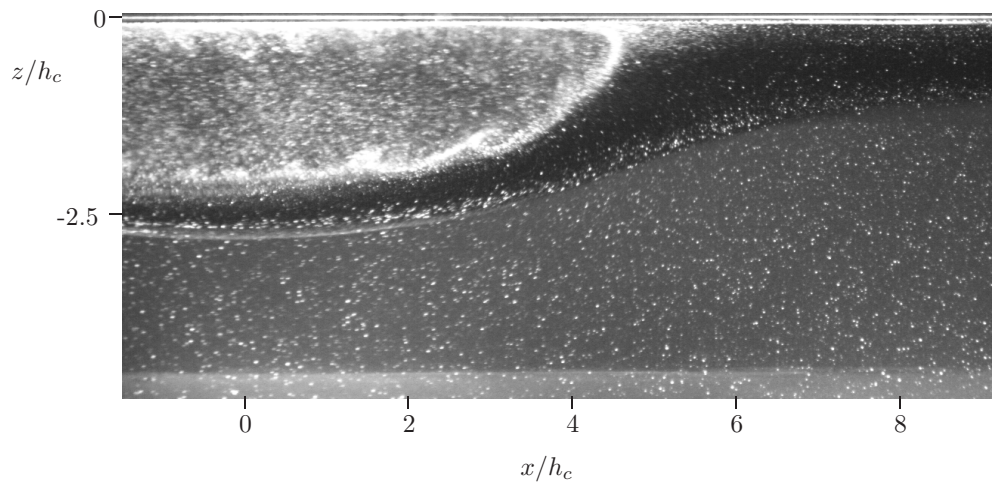

Figure 4: Frame 227 from experimental movie 24/04/06. 


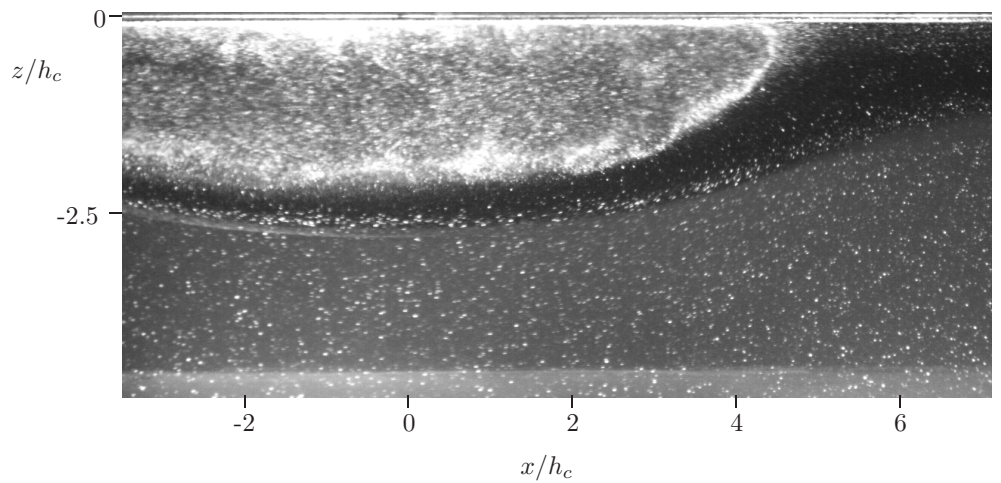

Figure 5: Frame 257 from experimental movie 24/04/06.

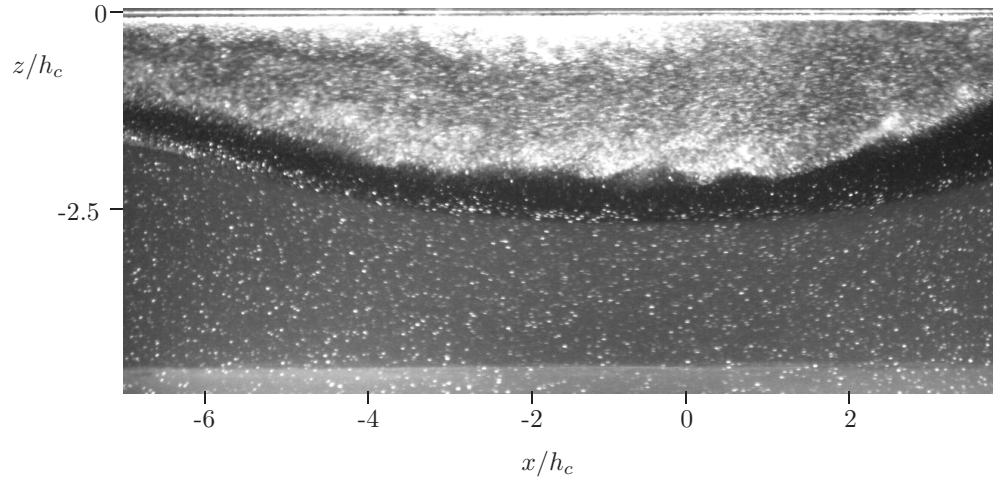

Figure 6: Frame 307 from experimental movie 24/04/06.

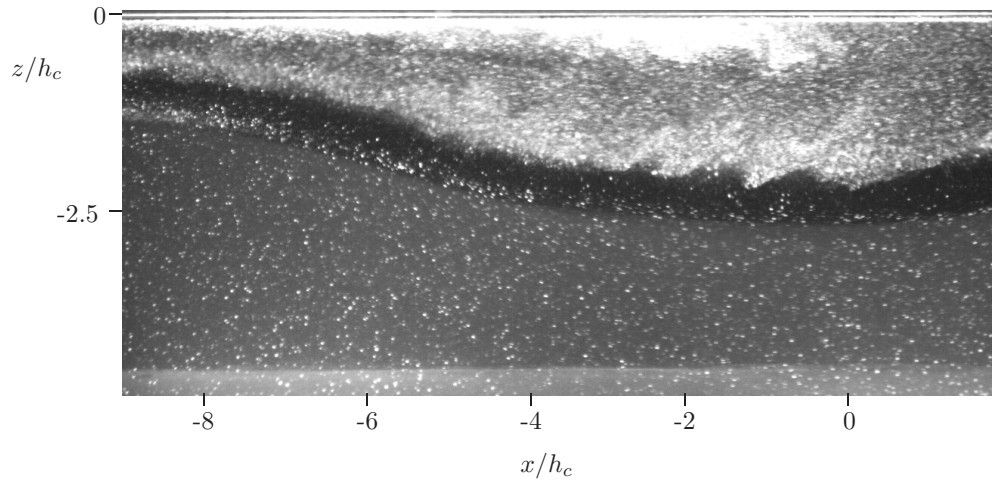

Figure 7: Frame 337 from experimental movie 24/04/06. 


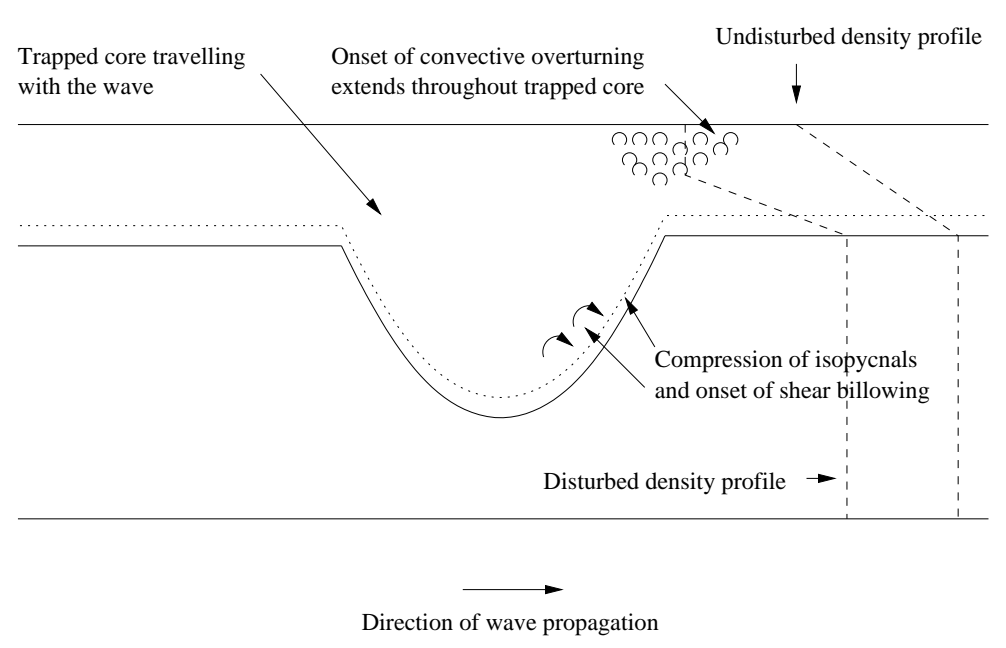

Figure 8: Schematic diagram of the unstable motion seen in the 2 layer regime.

where $\beta=-(\partial \rho / \partial z) / \rho$. It is well known that a steady shear flow is potentially unstable if $R i<1 / 4$ and stable if $R i>1 / 4$ (see Miles; ${ }^{15}$ Howard $^{16}$ ). The buoyancy frequency $g \beta$ is a measure of stabilizing effects while the velocity gradient $\partial u / \partial z$ is destabilizing. In a recent paper, Dalziel et al. ${ }^{17}$ have made simultaneous synthetic schlieren and PIV measurements of stable ISWs propagating in a three layer regime. They have shown that for a stable wave with undisturbed background $h_{3}=0.29, h_{2}=0.06$, and $h_{1}=0.02$, and non dimensionalised amplitude, $a^{*}=1.38$, that the vertical width of the pycnocline was compressed from $0.06 \mathrm{~m}$ in the undisturbed state to a width of approximately $0.04 \mathrm{~m}$ on the leading face. Note that Moum et al. ${ }^{2}$ also report a squeezing of isopycnals ahead of the trough (by a factor of 2). Fructus et al. ${ }^{9}$ have shown that ISWs in such a three layer configuration are stable even at very large amplitudes ( $a^{*}$ up to 1.74). In the two layer counterpart above however $\left(h_{3}=0.29 \mathrm{~m}, h_{2}+h_{1}=0.075 \mathrm{~m}\right)$, the flow is both shear and convectively - unstable at a non dimensional amplitude of $a^{*}=1.59$. Figure 4 (in particular) shows a compression of the isopycnals in the front half of the wave, similar to that documented in Dalziel et al. ${ }^{17}$ and Moum et al. ${ }^{2}$ The fundamental difference between the two cases is the presence of convective overturning in the two layer regime (it can be shown theoretically that convective instability is not possible in the three layer regime with 
a fixed upper boundary, see Fructus \& $\left.\mathrm{Grue}^{8}\right)$. It is conjectured that convective mixing in the top of the water column enhances isopycnal compression in the two layer regime. In addition, since the stratification is relatively weak and the convective overturning relatively vigorous, the stratification will tend toward a homogeneous state where convective mixing occurs. In effect, the two layer stratification will begin to look like that of the three layer regime but with enhanced isopycnal compression (see the dashed density lines suggested in Fig. 8). Enhanced isopycnal compression will increase $(\partial u / \partial z)^{2}$. This can be seen in Fig. 9 for experiment 24/04/06 where the variation in the horizontal velocity profile with distance $x / h_{c}$ from the trough is illustrated. Figure 9 can be compared directly with Fig. 4 . For $x / h_{c} \gtrsim 4.5$ the flow is stable. As the trough is approached $\left(x / h_{c}\right.$ diminishing), $\partial u / \partial z$ clearly increases $(\partial z / \partial u$ decreases $)$. In addition to an increase in $(\partial u / \partial z)^{2}$, an increase in the density gradient $\partial \rho / \partial z$ will accompany compression of the isopycnals. The effect of an increase in shear $(\propto(\partial u / \partial z))$ will be to destabilize the flow while an increase in the density gradient $(\partial \rho / \partial z)$ will be to stabilize it. However, with variation in $\partial z$, the destabilizing effect of $(\partial u / \partial z)^{2}$ is an order of magnitude greater than the stabilizing effect of $\partial \rho / \partial z$ (for constant $\partial u$ and $\partial \rho)$. Hence it is conjectured that shear instability is invoked in the front part of the wave as a result of enhanced isopycnal compression.

\section{B. Wave Motion}

Figure 10 shows a time series of the wave-induced horizontal velocity at different depths in the water column for a fixed $x$ location from the gate for experiment 24/04/06. For purposes of comparison with section IV, time $t$ is normalized by $\lambda / c$ where $c$ and $\lambda$ are the theoretically-computed speed and half width of the wave respectively. Details of how $c$ and $\lambda$ are computed can be found in Fructus et al. ${ }^{9}$ The horizontal velocity is nondimensionalized by $c$ and time is chosen to be zero when the interface is at maximum displacement. The front half of the wave is represented by negative $c t / \lambda$, as the time series goes from left to right. 


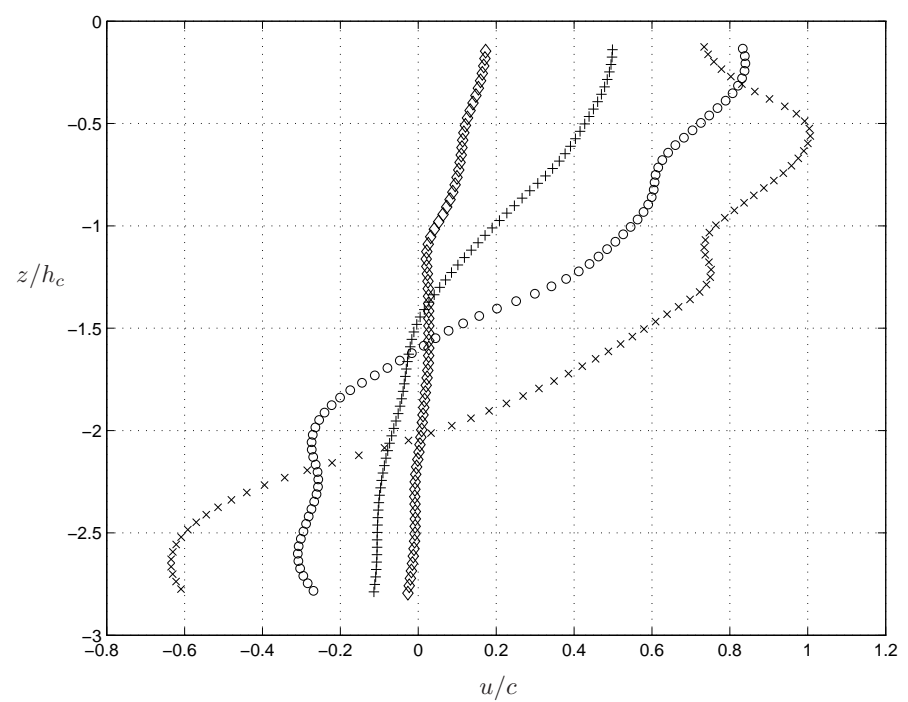

Figure 9: Depth $z / h_{c}$ versus horizontal velocity $u / c$ for experiment 24/04/06, frame 227 at $x / h_{c}=(\times) 2.0,(\circ) 4.5,(+) 6.0$, and $(\diamond) 8.0$.

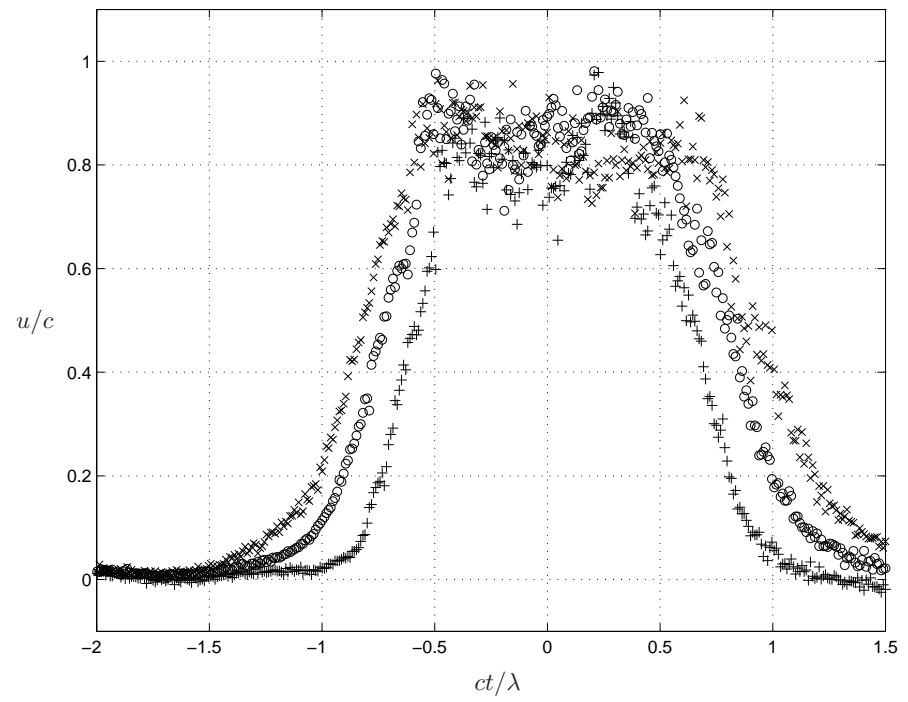

Figure 10: Horizontal velocity $u / c$ versus time $c t / \lambda$ for experiment $24 / 04 / 06$ at $z / h_{c}=$ $(\times)-0.51$, (o) -0.87 , and $(+)-1.23$. 
Figure 10 (and Figs. 11-13 below) illustrate the solitary wave character of the motion along the tank. Figure 10 clearly shows the initial manifestation of the instability in the wave at $c t / \lambda \approx-0.6$. Moreover, the increased turbulence associated with the development of the initial breaking is shown to result in increased scatter in the velocity data of Fig. 10 as $z / h_{c}$ becomes less negative (as the upper boundary is approached). Note that $u / c<1$, despite convective instability (small scale local overturning) being observed. This is investigated in more detail in section IV.

As the amplitude of the ISWs was reduced the vigor and extent of mixing in the top layer diminished (see Table I). A relatively small disturbance was seen in run 11/08/05 as a confined trapped core of fluid in the upper layer. The critical amplitude for instability when $h_{3}^{*} \approx 3.77$ (first data block) is $0.84 \pm 0.02<a_{\text {crit }}^{*}<1.07 \pm 0.02$ - a result that is in good agreement with the theoretical prediction of Fructus \& Grue ${ }^{8}$ of a critical amplitude of $a_{c r i t}^{*}=0.855$ for $h_{3}^{*}=4.13$ (see Fig. 13(a) in their work). Time series for experiments 11/08/05 (unstable) and 14/06/05 (stable) are given in Figs. 11 and 12 respectively. The amount and vertical extent of disturbance due to breaking is visibly less in Fig. 12 than Fig. 11 which, in turn, is less than in Fig. 10. Some scatter is seen at the top of the water column in Fig. 12. The wave in run 14/06/05 was stable, so a relatively smooth trace is expected for the velocity plot. The slight scatter is due to error in the PIV data; the experimental field of view was illuminated from below and it was difficult to eliminate all reflections from the underside of the upper boundary. In addition, tracer particles tended to congregate at the upper boundary adding to higher PIV error in this region. As a result it was difficult to gain very accurate data for $z / h_{c} \gtrsim-0.50$. Figure 13 shows the average horizontal velocity

$$
\bar{u}(t)=\int_{-1.24 h_{c}}^{-0.50 h_{c}} u(z, t) d z / 0.74 h_{c}
$$

as a time series for the three different experiments discussed above. The plot clearly shows 


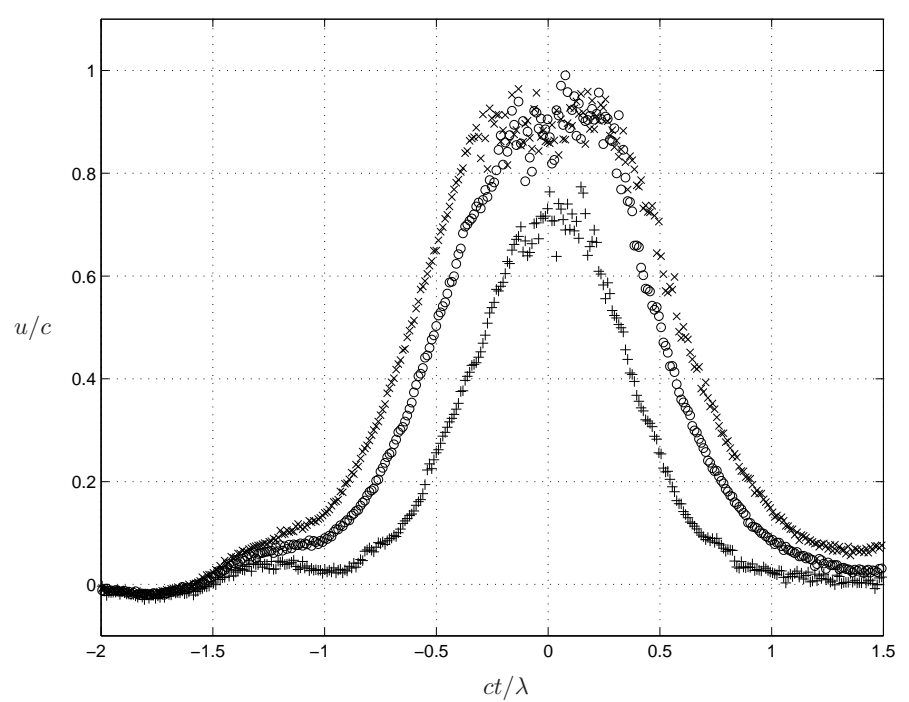

Figure 11: Horizontal velocity $u / c$ versus time $c t / \lambda$ for experiment $11 / 08 / 05$ at $z / h_{c}=$ $(\times)-0.51,(\circ)-0.87$, and $(+)-1.24$.

evidence of flow instability (increased data scatter) and wave width increase with wave amplitude.

In the second data block of Table I, $h_{3}^{*}$ is increased. In these experiments evidence of both shear and convective instability was seen but significant differences were noted from the corresponding plots in the first data block. Most notably, in run 26/04/06 shear instability was seen at the trough of the wave on the interface. This caused mixing and disturbance into the lower homogeneous layer from the trough to the tail. As the amplitude of the wave was reduced $(28 / 04 / 06)$ the overturning became less vigorous and more confined to the top layer, similar in nature to the behavior seen in the first data block. In the third data block, $h_{3}^{*}$ was increased further and the flow field disturbance resulting from instability was further enhanced. The vertical and horizontal extent of mixing into the bottom and top layers was more pronounced than in the previous blocks. The significant increase in instability and mixing from block 1 to 2 to 3 corresponds to an increase in $a_{\text {exp }}^{*}$ and $h_{3}^{*}$. In the last block, $h_{3}^{*}$ is increased once more. The breaking seen here was more violent than in the other cases, with a great deal of mixing in both the horizontal and vertical directions aft of the trough 


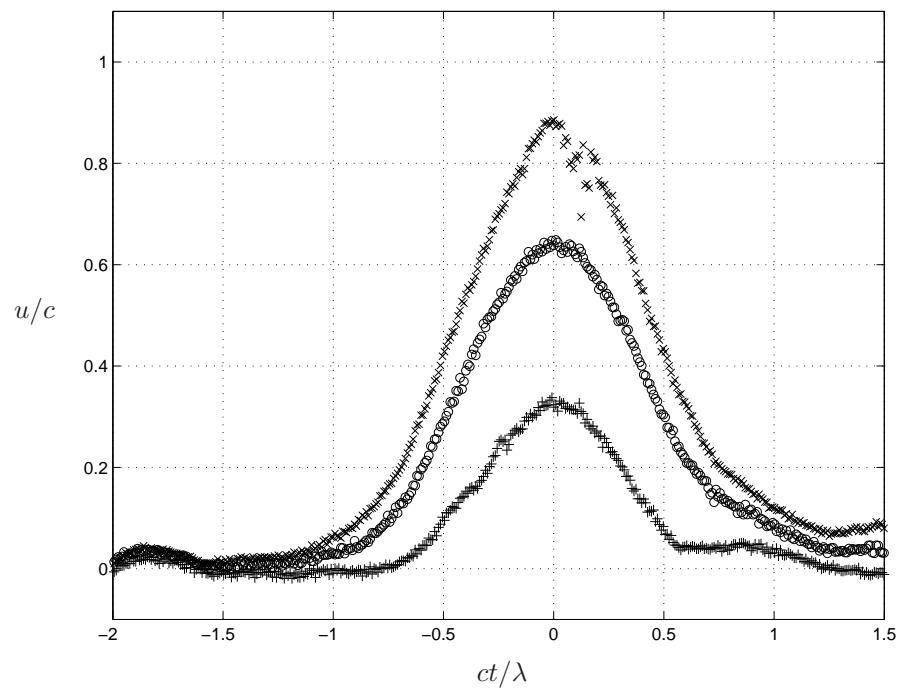

Figure 12: Horizontal velocity $u / c$ versus time $c t / \lambda$ for experiment $14 / 06 / 05$ at $z / h_{c}=$ $(\times)-0.50,(\circ)-0.85$, and $(+)-1.19$.

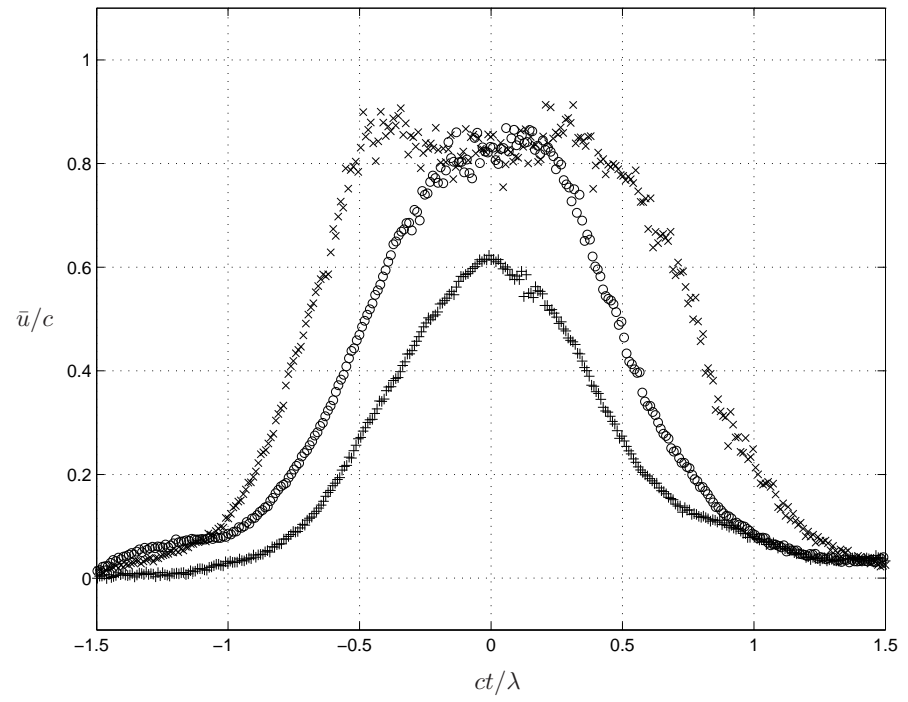

Figure 13: Average horizontal velocity $\bar{u} / c$ versus time $c t / \lambda$ for experiments $(\times)$ 24/04/06, (o) $11 / 08 / 06$, and $(+) 14 / 06 / 06$. 
and throughout the tail of the wave. Comparison of 12/08/05 with the first block implies an increase of $h_{3}^{*}$ stabilizes the flow for a fixed amplitude. In other words the larger $h_{3}^{*}$ the higher the critical amplitude $a_{c r i t}^{*}$. This is in agreement with the theoretical work of Fructus $\&$ Grue, ${ }^{8}$ who predict critical amplitudes of $a_{c r i t}^{*}=0.629,0.855$, and 0.971 for $h_{3}^{*}=2,4.13$, and 10 respectively, see Figs. 13(a) and 14 of their work. A word of caution is required here as there is also variation in $N$ between blocks 1 and 4 . Variation in $N$ does not change the fundamental stability characteristics of the problem but it does change the wave speed $c_{\exp }$ and hence the time scale of the waves being observed. The leading order change is to the linear and nonlinear wave speeds, $c_{0}$ and $c$ respectively; the ratio $c / c_{0}$ remains unchanged for a given wave amplitude (compare experiment 12/08/05 with 01/06/05, 23/06/05 with 26/04/05, and 20/07/05 with 01/05/06 in Table I).

In order to check the fundamental stability characteristics and resolution of the small tank a series of experiments were carried out on a larger scale using the bigger tank. The Brunt-Väisälä frequency was varied in these cases between $1.00 \mathrm{~s}^{-1}$ and $1.46 \mathrm{~s}^{-1}, h_{3}^{*}$ was varied from 4.85 to 6.19 and $a_{\text {exp }}^{*}$ from 1.86 to 2.28. Excellent agreement was found between the stability characteristics observed on the small scale and those on the larger scale. The larger scale experiments were performed using the same experimental apparatus as Grue et al. ${ }^{5}$ for a similar parameter range. Grue et al. ${ }^{5}$ however did not observe any evidence of shear instability in their observations. Table I suggests the amplitudes considered by Grue et al. ${ }^{5}$ were subcritical.

\section{Variation of the Upper Boundary Condition}

Table II. The effect of the upper boundary condition on the instability of an ISW in a 2 layered density stratification. 


\begin{tabular}{lcccccl}
\hline \hline Date & $h_{3}^{*}$ & $a_{\text {exp }}^{*}$ & $V(l)$ & $N\left(\mathrm{~s}^{-1}\right)$ & Boundary Condition & Instability Observed \\
\hline $11 / 04 / 05$ & 3.84 & $1.24 \pm 0.02$ & 59 & 1.49 & Free & Vigorous \\
$01 / 06 / 05$ & 3.81 & $1.63 \pm 0.03$ & 59 & 1.48 & Fixed & Moderate \\
$15 / 06 / 05$ & 3.87 & $1.45 \pm 0.03$ & 40 & 1.53 & Fixed & Slight-Moderate \\
$27 / 09 / 05$ & 3.92 & $1.49 \pm 0.03$ & 59 & 1.52 & Whetted & Moderate \\
\hline $07 / 04 / 05$ & 4.20 & $1.08 \pm 0.02$ & 40 & 1.57 & Free & Moderate \\
$11 / 08 / 05$ & 3.71 & $1.07 \pm 0.02$ & 29 & 1.50 & Fixed & Very Slight \\
\hline $14 / 04 / 05$ & 3.93 & $0.84 \pm 0.02$ & 30 & 1.52 & Free & Moderate \\
$14 / 06 / 05$ & 3.77 & $0.84 \pm 0.02$ & 20 & 1.55 & Fixed & None \\
\hline \hline
\end{tabular}

Table II illustrates the effect of the upper boundary condition on the stability of an ISW in a two layered density configuration. In the free surface case, 11/04/05, breaking took the same form as in the fixed cases $(01 / 06 / 05 \& 15 / 06 / 05)$ but was a lot more vigorous. Evidence of convective instability (small-scale mixing with $u>c$ ) appeared initially in the top of the water column and shear quickly developed on the leading face of the wave. Most of the mixing was confined to the top layer but extended lower than in the fixed counterparts. The interface was slightly disturbed at the trough but no significant mixing into the lower layer was seen. Moreover, comparison of 11/04/05 with 01/06/05 shows that fixing the free surface increases the amplitude of the wave by over $30 \%$ for a fixed generating volume $V$. Despite the increase in amplitude, instability is reduced significantly.

When the upper boundary is held fixed, free surface disturbance is eliminated and energy loss at the upper boundary is expected accordingly to be less than in the free case. This explains the increase in amplitude when a rigid lid and fixed generating volume are used. Imposing no slip at the upper boundary reduces dramatically the local fluid velocity within the vicinity hence less convective overturning is expected to be seen in the fixed case (as opposed to the free) even at larger amplitudes. Observation suggests that shear as well as convective mixing was reduced when the upper boundary was fixed, adding weight to the conjecture in section III A that the two instability types are intrinsically linked in this type of flow, such that convective instability induces shear.

In experiment 27/09/05, whetting agent was added to the free surface just prior to wave generation. Convective and shear instability were manifested but visibly less than in the un- 
whetted case (11/04/05) and visibly more than in the fixed cases (01/06/05 and 15/06/05).

Grue et al. ${ }^{5}$ postulate that surface tension plays a role in convective instability. It is shown here (and later in Tables III \& IV) that surface tension does indeed aid, or induce convective mixing. Finally the last four experiments in Table II provide confirmation that fixing the free surface reduces or eliminates convective (and hence shear) instability in this regime.

Table III. The effect of the upper boundary condition on the stability of an ISW in a 3 layer density stratification consisting of homogeneous top and bottom layers with a sharp pycnocline in between.

\begin{tabular}{|c|c|c|c|c|c|c|c|c|}
\hline Date & $h_{3}^{*}$ & $h_{2} / h_{1}$ & $a_{e x p}^{*}$ & $V(l)$ & $N$ & Boundary Condition & Shear & Convective \\
\hline $25 / 05 / 05$ & 7.93 & 1.89 & $2.56 \pm 0.06$ & 60 & 3.11 & Free & Vigorous & Vigorous \\
\hline $31 / 05 / 05$ & 7.56 & 1.44 & $3.30 \pm 0.07$ & 60 & 3.20 & Fixed & Vigorous & None \\
\hline $19 / 07 / 05$ & 6.98 & 1.21 & $2.57 \pm 0.06$ & 40 & 3.25 & Fixed & Vigorous & None \\
\hline $26 / 09 / 05$ & 7.38 & 1.67 & $2.75 \pm 0.06$ & 60 & 3.03 & Whetted & Vigorous & Moderate \\
\hline $27 / 05 / 05$ & 4.13 & 0.73 & $1.50 \pm 0.03$ & 60 & 2.80 & Free & Slight & Slight \\
\hline $20 / 06 / 05$ & 4.00 & 0.76 & $1.59 \pm 0.04$ & 60 & 2.73 & Fixed & Moderate & None \\
\hline $13 / 06 / 05$ & 4.41 & 0.42 & $1.56 \pm 0.03$ & 60 & 3.46 & Fixed & Moderate & None \\
\hline
\end{tabular}

To clarify these concepts it is worth considering instability in the three layer regime. A detailed investigation of the instability of ISWs in a three layered density configuration with a fixed upper boundary can be found in Fructus et al. ${ }^{9}$ For purposes of comparison, experiments 31/05/05, 19/07/05, 20/06/05 and 13/06/05 from Fructus et al. ${ }^{9}$ are briefly repeated here. Table III illustrates the effect of the upper boundary condition in the three layer configuration. The upper and lower layers are homogeneous and of depth $h_{1}$ and $h_{3}$ respectively. The pycnocline is relatively sharp, linearly stratified and of depth $h_{2}$ (see Fig. 1(b)). In the free case, 25/05/05, vigorous overturning resulting from shear and convective instability was seen. Convective mixing (small-scale overturning) started in the top of the water column, near the free surface at the front of the wave, $\left(x^{*}, z^{*}\right) \approx(10,-0.2)$ in Fig. 14. Note it is difficult to see this although particles do appear slightly out of focus here due to the mixing. This continued to develop with the passage of the wave as overturning throughout the top layer. Billowing started on the interface at the trough of the wave as a result of shear instability and rapidly developed into turbulence in the tail mixing both the top and bottom layers (see Figs. $14 \& 15$ ). There did not appear to be interaction 


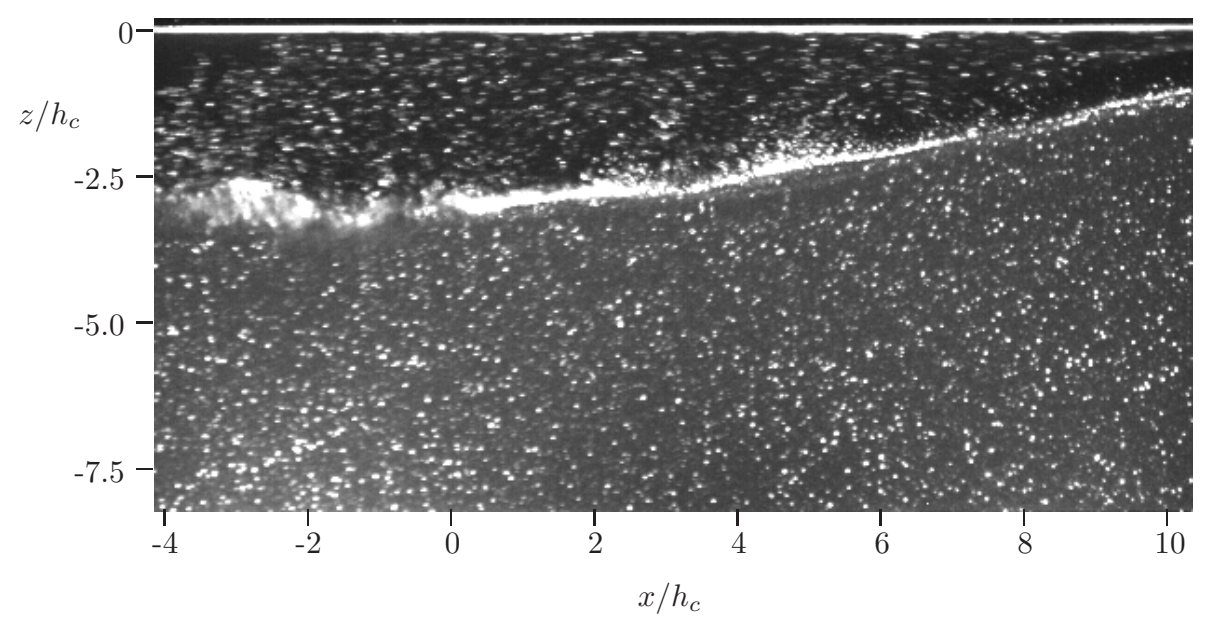

Figure 14: Frame 317 from experimental movie 25/05/05. Free surface induced convective instability began at $\left(x^{*}, z^{*}\right) \approx(10,-0.2)$ and developed throughout the front, top part of the wave. Shear instability began on the interface at approximately the trough.

between the flow components associated with the two instability types. Comparison of 25/05/05 with 31/05/05 shows again that fixing the free surface increases the amplitude of the wave by nearly 30\% (in this case for a fixed generating volume $V$ ). Moreover all convective instability was eliminated in the fixed case and shear instability was observed to be enhanced significantly (see Fig. 16). Observation of experiments 25/05/05 and 19/07/05 suggest that the upper boundary condition has no effect on shear instability and confirms that there is no interaction between the shear and convective modes seen in 25/05/05. On the other hand, the effect of the upper boundary condition on convective instability is quite clear and profound. With a fixed surface, convective instability is eliminated completely (cf. 25/05/05 with 31/05/05 and 19/07/05). Comparison of the last three experiments in Table III confirms this and also shows an increase in amplitude and shear for a rigid lid and a fixed generating volume.

It can be shown by conservation of mass that in a three layer density configuration in which the top layer is homogeneous convective breaking is not possible (see Fructus \& Grue $^{8}$ ). Therefore, the convective instability observed here must be associated with the up- 


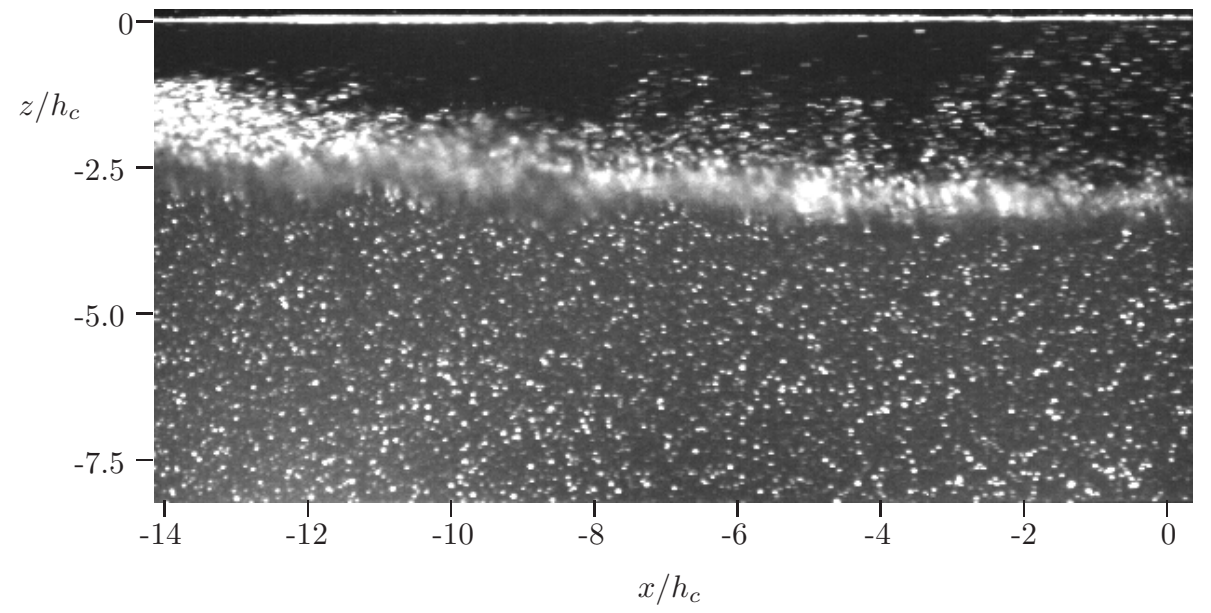

Figure 15: Frame 398 from experimental movie 25/05/05. Vigorous mixing in the tail of the wave.

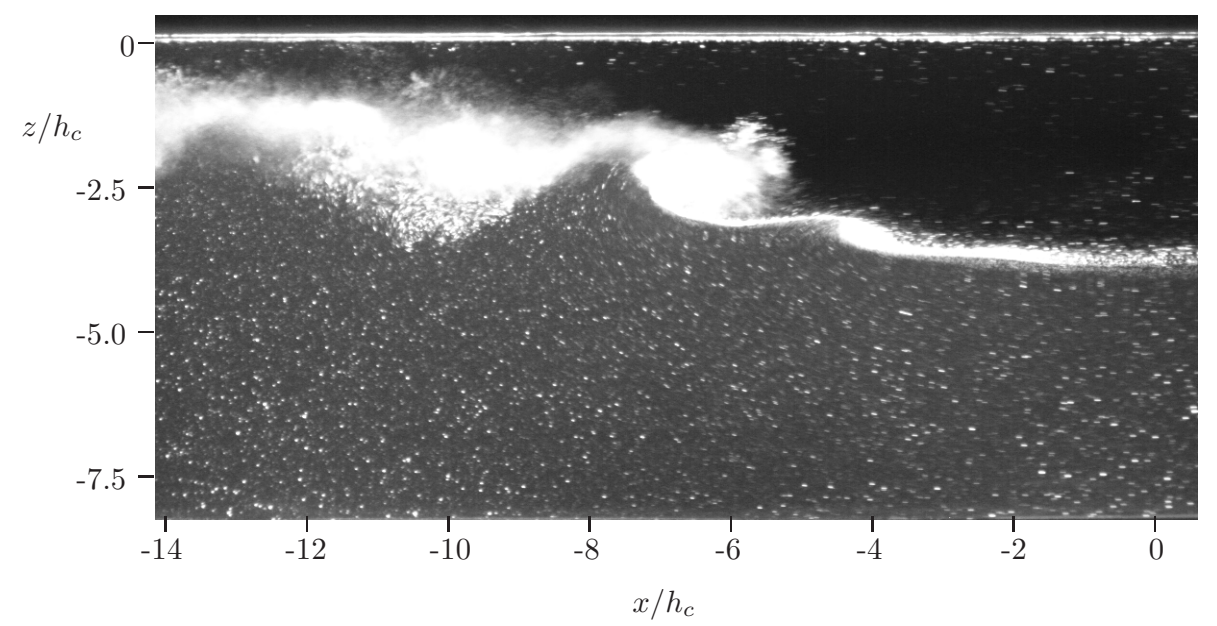

Figure 16: Frame 344 from experimental movie 31/05/05. Vigorous mixing in the tail of the wave. 
per boundary condition. In the whetted case, 26/09/05, convective instability was present but visibly less than in the un-whetted case, 25/05/05. This suggests further that the upper boundary condition and, in particular, surface tension can play a crucial role in the onset of convective instability.

Table IV. The effect of the upper boundary condition on the instability of an ISW in a 3 layer density stratification consisting of homogeneous top and bottom layers with a diffuse pycnocline in between.

\begin{tabular}{|c|c|c|c|c|c|c|c|}
\hline Date & $h_{3}^{*}$ & $h_{2} / h_{1}$ & $a_{e x p}^{*}$ & $V(l)$ & $N$ & Boundary Condition & Instability Observed \\
\hline $13 / 04 / 05$ & 4.23 & 3.38 & $1.50 \pm 0.03$ & 58 & 1.49 & Free & Moderate; Combination \\
\hline $02 / 06 / 05$ & 4.16 & 4.07 & $1.73 \pm 0.04$ & 58 & 1.48 & Fixed & None \\
\hline $06 / 06 / 05$ & 4.21 & 2.89 & $1.53 \pm 0.03$ & 42 & 1.50 & Fixed & None \\
\hline $28 / 09 / 05$ & 3.97 & 2.95 & $1.53 \pm 0.03$ & 58 & 1.43 & Whetted & Slight; Combination \\
\hline $28 / 06 / 05$ & 3.28 & 2.26 & $1.24 \pm 0.03$ & 58 & 1.38 & Free & Slight; Convective \\
\hline $27 / 06 / 05$ & 3.20 & 1.69 & $1.20 \pm 0.03$ & 58 & 1.46 & Free & Slight; Convective \\
\hline $01 / 07 / 05$ & 3.33 & 1.90 & $1.43 \pm 0.03$ & 58 & 1.41 & Fixed & None \\
\hline
\end{tabular}

Table IV provides further insight. In this instance, the background stratification consists of a linearly-stratified, diffuse pycnocline $\left(h_{2} / h_{1}\right.$ larger than in Table II) sandwiched between two homogeneous layers (see Fructus et al. ${ }^{9}$ ). The table shows no evidence of instability when the upper boundary is fixed. The instability occurring in the free-surface case appeared to be a combination of shear and convective types, beginning toward the front of the wave. The fact that all instability is eliminated in the rigid lid case implies the two modes of instability interact. If the interaction is such that convective instability acts to enhance shear, as conjectured in III A, then this explains why an elimination of shear is seen when the free surface is fixed. Comparison of 13/04/05 and 28/09/05 provides evidence that whetting the free surface reduces the magnitude of overturning, implying again that convective instability is somewhat surface tension-induced. Comparison of the last three experiments in the table confirm the earlier result that a rigid lid eliminates (or reduces) convective overturning. 


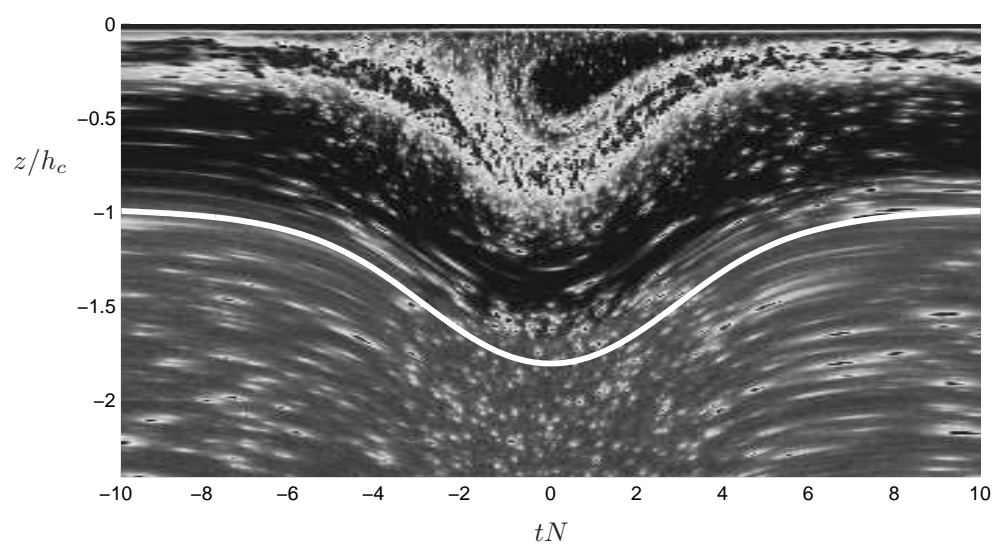

Figure 17: Reconstructed wave profile from experiment 14/06/05 and the corresponding fully nonlinear numerical solution (thick white line)

\section{THEORETICAL DEVELOPMENT}

Fructus \& Grue $^{8}$ developed a fully nonlinear numerical model of ISW propagation in a stably stratified layered fluid. In Fructus et al. ${ }^{9}$ the nonlinear model of Fructus \& Grue ${ }^{8}$ was developed and compared with data from experimentally-generated ISWs in the three layer regime. Strictly speaking, the theory is valid for waves with induced velocities less than or equal to the wave speed $c$. Beyond that the assumption of the constant density lines decaying with the vertical coordinate is broken in the trapped core of the computational wave. For stable (i.e. non-breaking) waves, the fully nonlinear model and experimental data showed excellent agreement. In the unstable cases significant discrepancies were found. The numerical model provides a stable solution to the fully nonlinear internal wave equations so disagreement was expected in the experimental cases where instability was in evidence. A comparison for the two layer regime is considered here. Experiment 14/06/05 is the only example in which the wave was stable and in which (i) good agreement is expected to be seen between theory and experiment and (ii) the theory is strictly valid. The background in Fig. 17 shows the changes with time of the pixel values in a given column of the experimentally-captured digitized image for 14/06/05. The background image was generated using DigiFlow's time- 
series function. The superimposed solid white line is a trace of the interface given by the fully nonlinear solution for exactly matched initial conditions. There is excellent agreement between the shape of the two traces. In Fig. 17 the front of the wave is the left half of the trace as it is a time series of wave propagation. Figure 18 shows the variation in a) velocity, $u / c$ and b) vorticity, $\omega h_{c} / c$ with depth, $z / h_{c}$, at the trough of the wave. $c$ is the computed wave speed and $h_{c}=h_{1}+h_{2}$. The solid black line refers to the theoretical result and the crosses to the experimental PIV data. Excellent agreement is seen between the two sets of data. Note in this instance the wave is convectively stable and $u / c<1$. A similar set of figures for 11/08/05 are shown in Figs. 19 and 20. In this instance there was only slight disturbance in the experimental wave and good agreement is seen between the two data sets (despite the theory not being strictly valid). Excellent agreement is found for the interfacial traces implying that the shape of the wave is not affected by relatively weak mixing confined to a small region well above the interface. Slight discrepancy is seen between the theoretical and experimental data in the velocity (and vorticity) profiles toward the top of the water column, a result of the experimental wave being slightly unstable there. Note also that $u / c$ attains a value of 1 in the upper part of the water column, in both the numerical and experimental data, confirming convective instability is present in the laboratory simulations. Recall that strictly speaking the numerical data is only valid for values of $u$ up to $c$. In the very top of the water column the experimental value of $u / c$ tends to zero. This is a result of the upper boundary being fixed. In run 24/04/06 a significant amount of mixing is seen in the experimental wave (see Table I). This is captured in Figs. 21 and 22 where significant discrepancy is seen between the theoretical and experimental data sets. The shape of the wave is affected directly by the instability. The interface was not disturbed but a broadening of the wave, like that reported in Grue et al.,,$^{5}$ is clearly seen. Note the enhanced isopycnal compression in the front of the wave (left hand side of Fig. 21). The experimental data shows $u / c<1$ (although $u$ is very close to $c$ at $z / h_{c}=-0.9$ ) despite convective instability 

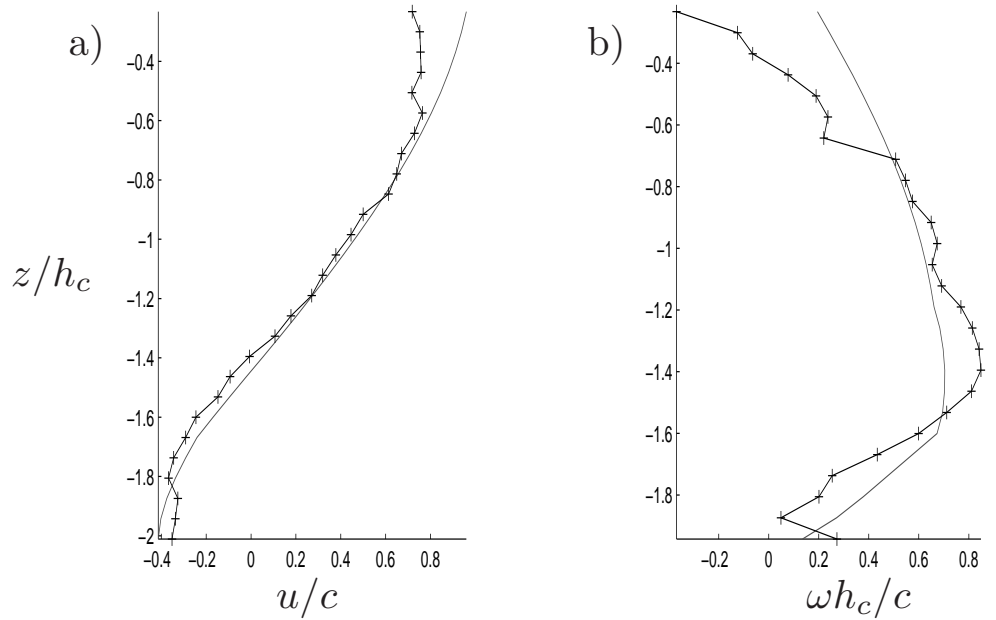

Figure 18: a) measured $(+)$ and computed (solid line) velocity profile for 14/06/05. b) measured $(+)$ and computed (solid line) vorticity profile for 14/06/05.

being observed (cf. Fig. 10). This is a result of the disturbed flow being well developed by the measurement location, a fuller explanation of this is given later. 


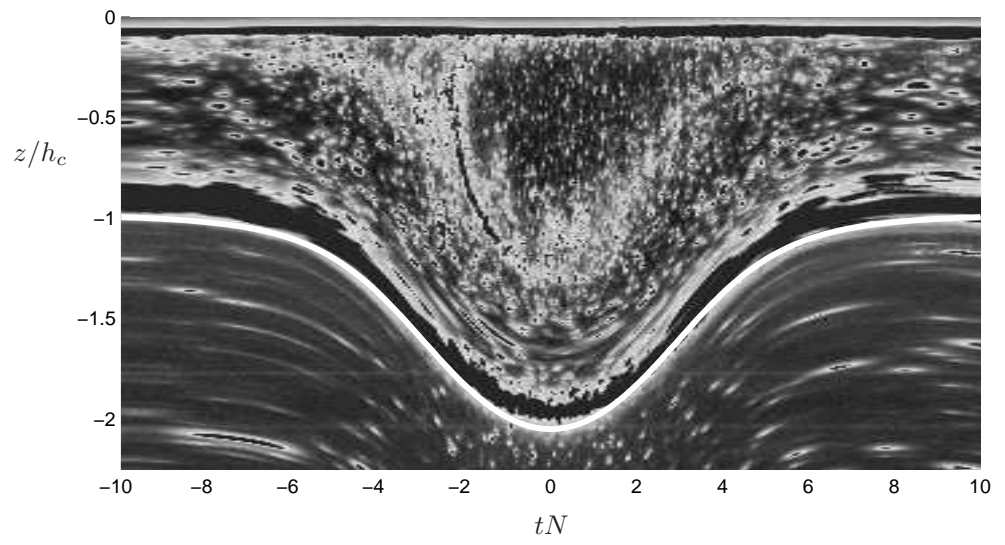

Figure 19: Reconstructed wave profile from experiment 11/08/05 and the corresponding fully nonlinear numerical solution (thick white line)
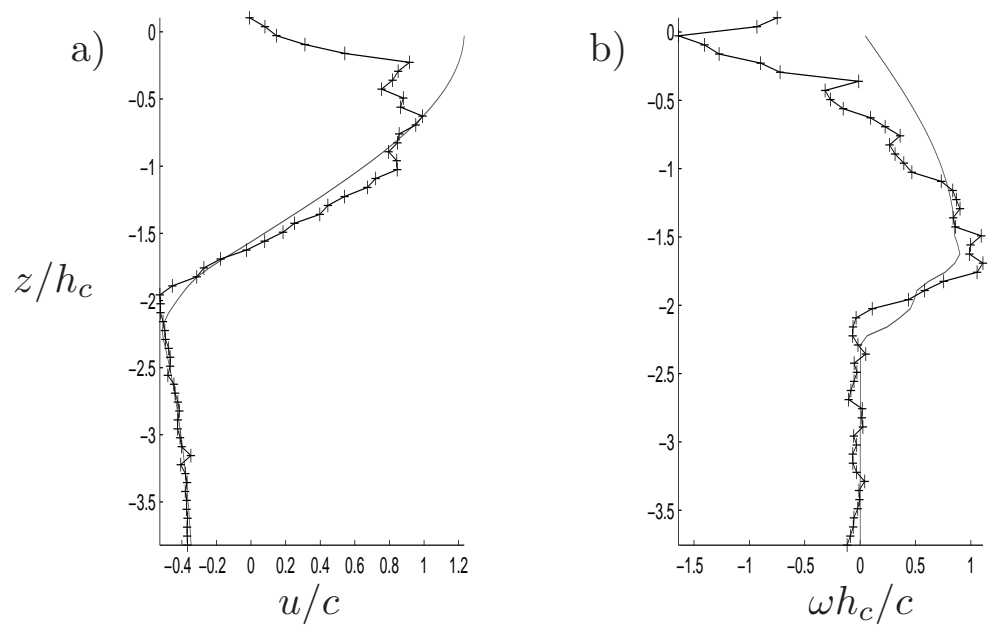

Figure 20: a) measured $(+)$ and computed (solid line) velocity profile for 11/08/05. b) measured $(+)$ and computed (solid line) vorticity profile for 11/08/05. 


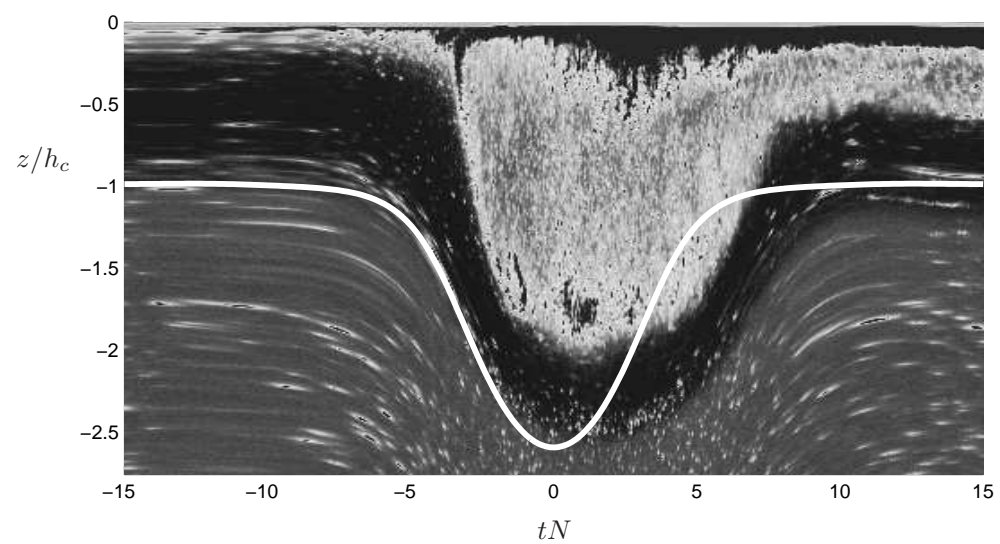

Figure 21: Reconstructed wave profile from experiment 24/04/06 and the corresponding fully nonlinear numerical solution (thick white line)

The theoretical model can be used to predict the threshold amplitude $a_{\text {crit }}$ for breaking, with $u / c=1$, at a single point. For larger amplitudes, the model predicts that $u / c>1$ at the top of the water column, in the trapped core. The theory is not strictly valid once $u>c$, nevertheless, a map of the region in which $u / c>1$ can be computed to mark an expected convectively unstable region. In addition the value of the Richardson number throughout the wave domain can be computed, see Fructus et al. ${ }^{9}$ Such maps for 11/08/05 and 24/04/06 are given in Figs. 23 and 24 respectively. The shaded area marks the convectively unstable region in which $u / c>1$ and the grey contour line refers to $R i=0.25$. Both figs show a region in the upper part of the wave in which $u / c>1$ and $R i<1 / 4$. This implies that the fully nonlinear solution is expected to be convectively unstable and potentially, though not necessarily, unstable with respect to shear. The experimental observations confirm this prediction, as convective instability was seen in both cases and shear instability was evident in run 24/04/06. Fructus et al. ${ }^{9}$ have shown that shear instability in ISWs is parametrized not by the smallest $R i$ in the flow but rather by the horizontal extent of $R i \leq 0.25$ (denoted by $L_{x}$ ), divided by the half width, $\lambda$. The parameter $L_{x} / \lambda$ takes the value 0.78 and 0.51 for experiments $24 / 04 / 06$ and $11 / 08 / 05$ respectively, indicating that shear in 11/08/05 is significantly less than in 24/04/06. The horizontal extent of the unstable region with respect 

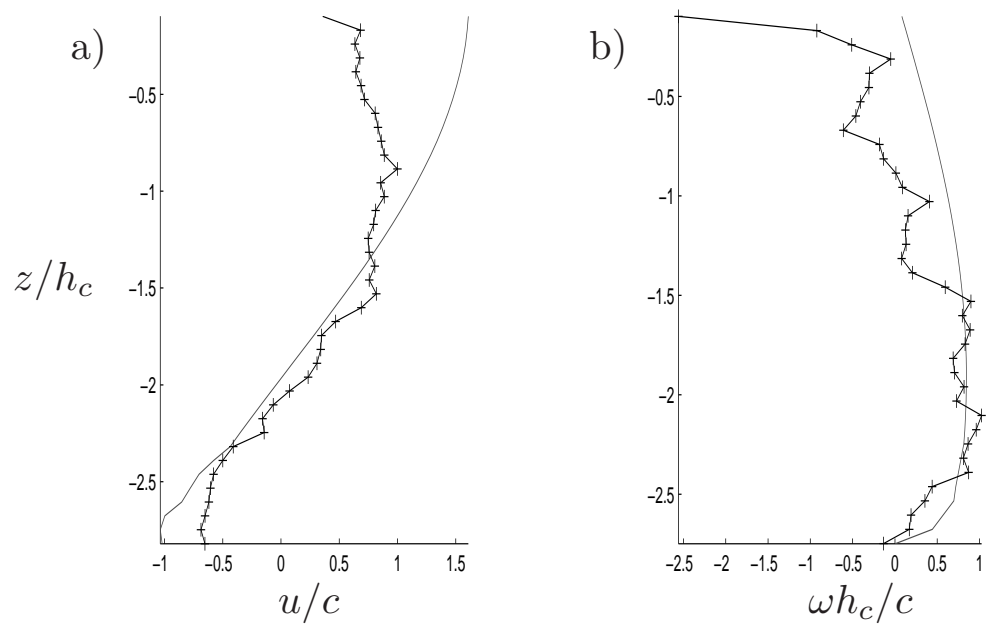

Figure 22: a) measured $(+)$ and computed (solid line) velocity profile for 24/04/06. b) measured $(+)$ and computed (solid line) vorticity profile for 24/04/06.

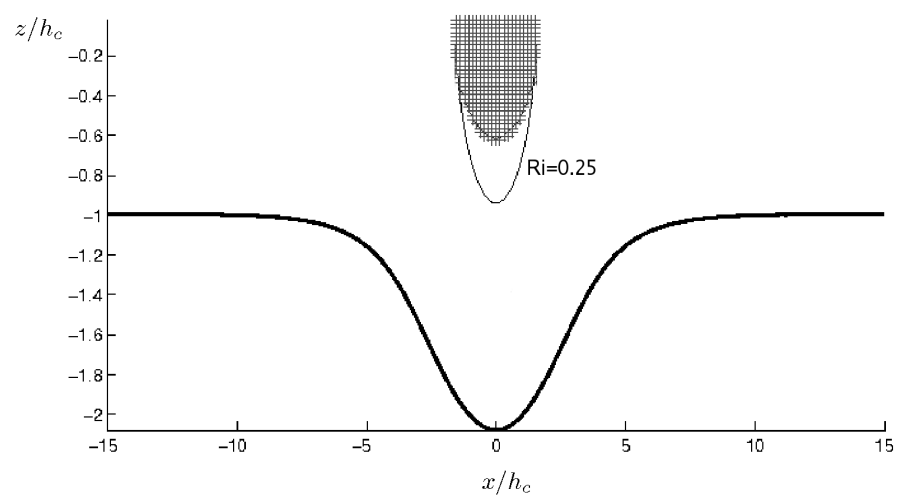

Figure 23: Fully nonlinear solution for 11/08/05. The shaded area marks the convectively unstable region in which $u / c>1$, the light grey contour marks $R i=0.25$, and the thick black line indicates the interface. 


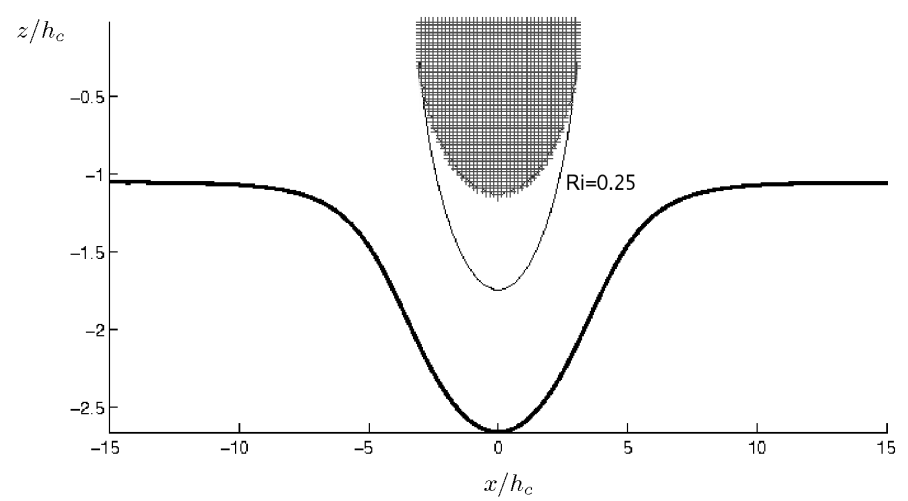

Figure 24: Fully nonlinear solution for 24/04/06. The shaded area marks the convectively unstable region in which $u / c>1$, the light grey contour marks $R i=0.25$, and the thick black line indicates the interface.

to convective instability can be parametrized in a similar fashion. Let $w_{x}$ denote the half width of the theoretically-computed region $u / c=1$ at the top of the water column. Then $w_{x} / \lambda$ takes the values 5.25 and 3.29 for experiments $24 / 04 / 06$ and 11/08/05 respectively. $w_{x} / \lambda$ is significantly bigger in 24/04/06 reflecting the difference in prevalence and vigor of convective instability seen between the two cases.

Table V. A comparison of the experimental and theoretical data.

\begin{tabular}{ccccccccc}
\hline \hline Date & $h_{3}^{*}$ & $a_{\text {exp }}^{*}$ & $a_{\text {theo }}^{*}$ & $c_{\text {exp }}$ & $c_{\text {theo }}$ & $x_{\text {exp }} / \lambda$ & $x_{\text {theo }} / \lambda$ & $(u / c)_{\max }$ \\
\hline $01 / 06 / 05$ & 3.81 & $1.63 \pm 0.03$ & 1.63 & $0.115 \pm 0.002$ & 0.13 & 0.67 & 0.41 & 0.60 \\
$24 / 04 / 06$ & 3.87 & $1.59 \pm 0.03$ & 1.66 & $0.111 \pm 0.002$ & 0.13 & 0.60 & 0.39 & 0.80 \\
$15 / 06 / 05$ & 3.87 & $1.45 \pm 0.03$ & 1.52 & $0.108 \pm 0.002$ & 0.13 & 0.41 & 0.37 & 0.80 \\
$25 / 04 / 06$ & 3.59 & $1.23 \pm 0.02$ & 1.26 & $0.098 \pm 0.002$ & 0.11 & 0.41 & 0.33 & 1.00 \\
$11 / 08 / 05$ & 3.71 & $1.07 \pm 0.02$ & 1.08 & $0.104 \pm 0.002$ & 0.11 & 0.25 & 0.25 & 1.00 \\
$14 / 06 / 05$ & 3.77 & $0.84 \pm 0.02$ & 0.85 & $0.095 \pm 0.001$ & 0.10 & 0.00 & 0.00 & NA \\
& & & & & & & & 0.36 \\
$26 / 04 / 06$ & 5.65 & $2.44 \pm 0.05$ & 2.57 & $0.093 \pm 0.001$ & 0.11 & 1.06 & 0.36 & 0.35 \\
$28 / 04 / 06$ & 5.27 & $1.97 \pm 0.04$ & 1.93 & $0.093 \pm 0.001$ & 0.10 & 0.62 & 0.38 & 0.70 \\
$27 / 04 / 06$ & 5.41 & $1.16 \pm 0.03$ & 1.16 & $0.076 \pm 0.001$ & 0.08 & 0.30 & 0.27 & 0.75 \\
\hline \hline
\end{tabular}

Table $\mathrm{V}$ gives a comparison of the experimentally-measured and theoretically-computed critical parameters of interest. There is remarkably good agreement between the measured and computed wave speeds and amplitudes, even in the unstable cases (14/06/05 is the only stable wave). Here, $x_{\text {theo }}\left(=w_{x}\right)$ is the horizontal distance from the trough of the wave to the point at which $u / c=1$ computed theoretically. The quantity $x_{\text {theo }}$ is valid up to the point of 
breaking and is a measure for the point of onset of convective instability while $x_{\exp }$ is taken from the experimental movie and is a subjective estimate of the horizontal distance from the trough of the wave to the point at which convective instability is seen for a given frame. For run 24/04/06 probing of the experimental movie yielded $x_{\text {exp }} / \lambda=0.60$. This is in agreement with the PIV data presented in Fig. 10 which shows instability beginning at $c t / \lambda \approx-0.60$ (which transforms to $x / \lambda=0.60$ ) for $z / h_{c}=-0.51$. Table $\mathrm{V}$ shows a significant discrepancy exists between $x_{\text {exp }} / \lambda$ and $x_{\text {theo }} / \lambda$. Moreover this discrepancy increases with wave amplitude. This is due to the stage at which instability is observed experimentally. For example, in a large amplitude case (such as 24/04/06) instability occurs well before the viewing location. By the time the wave reaches the measurement station the instability has grown and is well developed. Experimental observation is not of the onset of instability and as a result it is expected to be different from that predicted theoretically. Discrepancy between the two sets of results is larger for larger amplitude waves as instability grows with amplitude. $(u / c)_{\max }$ is the maximum value of $u / c$ measured at $x_{\exp }$. From Figs. 4 and $9,(u / c)_{\max }$ is of the order 0.80 for experiment 24/04/06 (maximum value of $u / c$ attained when $x / h_{c}=4.5$ ). Table $\mathrm{V}$ shows that $(u / c)_{\max }$ is smaller for larger amplitude cases. The fact that $(u / c)_{\max }$ decays with increasing amplitude further reflects the fact that instability develops with amplitude and the dynamics are not what may have been expected. Note that the wave speed $c$ in the above discussion is theoretically computed and constant. In practice the wave speed varies with wave propagation. In Grue et al. ${ }^{5}$ a locally computed wave speed was used to estimate $u / c$ and it was found that $u / c \approx 1$ in their convectively unstable cases. A similar computation is not possible here due to the extent of breaking. The fact that $c$ varies (and decays) with wave propagation offers an alternative explanation for $u / c \neq 1$ in the unstable cases. Additionally, in the experiments performed here a fixed upper boundary condition was imposed, so $u / c$ is expected to tend to zero in the very top of the water column, in contrast to the experiments of Grue et al. ${ }^{5}$ in which the upper boundary was free and hence there was no boundary layer. 


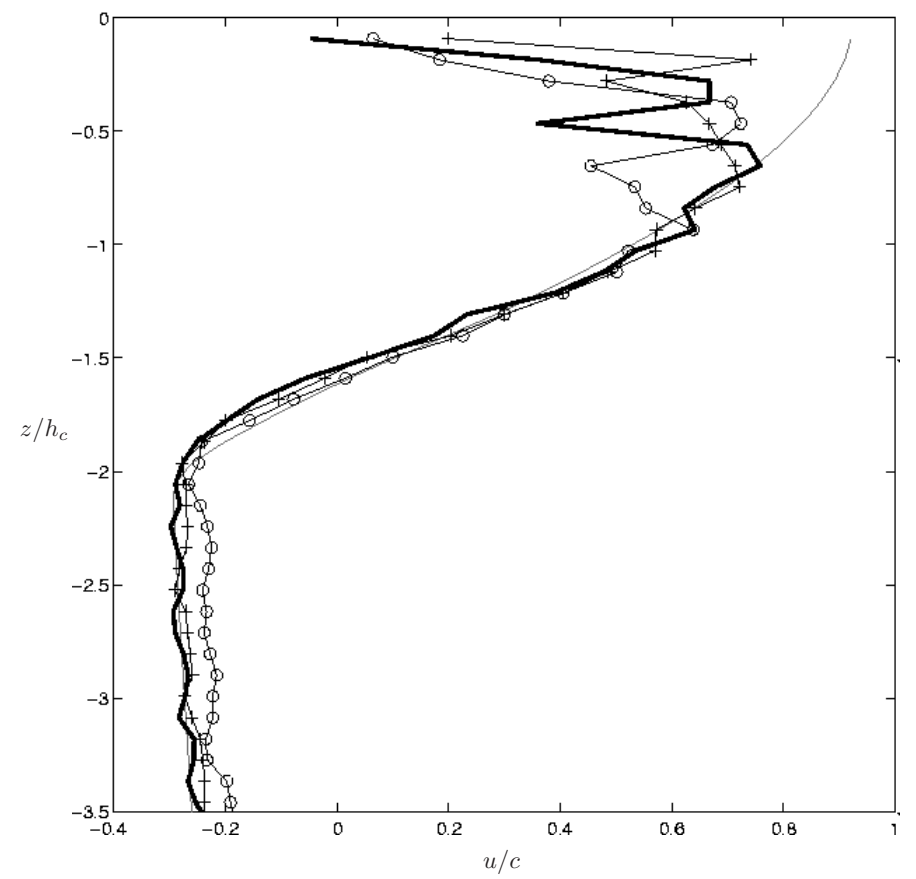

Figure 25: Measured velocity profile for 27/04/06 at frames: 270 (thick solid line), $372(+)$ and 461 (o), against theoretical computation (thin solid line).

In Fig. 25 an example of how developing instability may affect the velocity profile of an ISW is given. The plot is taken from 27/04/06 and shows the velocity profile at the trough of the wave at different time intervals throughout the waves development. The experimental data were captured at 24 frames per second. For $z / h_{c} \leq-0.7$ there is good agreement between the theoretical (thin line) and first set of experimental data (frame 270, thick line). As time progresses (frames 372 and 461) discrepancy between the theoretical (steady state solution) and experimental data increases. This suggests that the disturbance to the flow, a result of instability, increases with time. A full investigation of the growth and decay of ISW-induced instabilities is beyond the scope of the present paper. 


\section{COMPARISON WITH FIELD OBSERVATIONS}

The instability manifested in the leading part of Fig. 4 is similar in nature to that revealed in field observations made by Moum et al. ${ }^{2}$ (see Figs. $5 \& 14$ of their work). Moum et al. ${ }^{2}$ present evidence of Kelvin-Helmholtz-like billows growing from instabilities on the forward face of a strongly nonlinear IW. The instability in their observations was also confined to the top layer with little or no disturbance into the lower homogeneous water column. Moum et al. ${ }^{2}$ suggest that the instability responsible for their observed flow is like that reported in Grue et al. ${ }^{5}$ and argued that the instability they (and Grue et al..$^{5}$ ) observed was a result of small scale straining. The results of section III B imply that the instability seen in Grue et al. ${ }^{5}$ is strongly induced by the free surface and hence different from that seen in Moum et al. ${ }^{2}$ where surface tension effects are thought to be negligible. The normalized amplitude of the unstable wave sampled by Moum et al. ${ }^{2}$ is approximately 1.45 , more comparable to those amplitudes studied here than by Grue et al. ${ }^{5}$ The maximum velocity in the waves observed by Moum et al. ${ }^{2}$ was close to the wave propagation speed. In light of these comparisons and the new experimental data presented above it is possible that the disturbance seen in Moum et al. ${ }^{2}$ is a combination of shear and convective instability.

The background stratification in Moum et al. ${ }^{2}$ is similar to that considered here but not identical. In addition the background flow in the laboratory is quiescent whereas in the field it is not. Statsna \& Lamb ${ }^{18}$ show numerically that the presence of a non-constant background current has a strong effect on the stability of large, fully nonlinear ISWs. In particular, ISWs may break in the presence of a background current when they would not have done so otherwise for a given stratification. This may offer an alternative or supplementary explanation for the shear instability observed in Moum et al. ${ }^{2}$ An exact comparison between the field observations and experimental results must be made with caution. It has been shown how sensitive instability is to both background stratification and the upper boundary 
condition and this must be kept in mind when drawing comparisons.

\section{SUMMARY AND CONCLUSIONS}

The stability characteristics of an ISW in a two-layer density configuration consisting of a lower homogeneous layer and a linearly-stratified top layer, have been investigated both experimentally and theoretically. It was found that instability may manifest itself as a combination of shear and convective instability on the leading face of the wave. Moreover shear instability was shown to be induced by the development of convective instability higher in the water column. It is conjectured that convective instability enhances isopycnal compression in the front half of the wave leading to a reduced value of the local Richardson number there and consequential shear instability. The general stability characteristics inferred experimentally showed good agreement with the theoretical predictions of Fructus \& Grue. ${ }^{8}$ In particular it was shown that the depth ratio $h_{3}^{*}=h_{3} /\left(h_{1}+h_{2}\right)$ stabilized the flow and the critical amplitude for instability was $0.84 \pm 0.02<a_{c r i t}^{*}<1.07 \pm 0.02$ for $h_{3}^{*}, c$ and $N$ of the order $3.77,0.11 \mathrm{~ms}^{-1}$ and $1.5 \mathrm{~s}^{-1}$ respectively (see section III A).

As well as the form of the background stratification, the upper boundary condition had a significant effect on wave breaking conditions and propagation properties of ISWs in shallow water. In particular conditions for convective instability and the dependence upon wave amplitude were directly affected. It was shown that capillarity effects at the free surface can induce significant instability in an experimentally-generated wave that is otherwise stable (compare the last two experiments of Table II, for example). Moreover, it was possible to generate much larger amplitude waves for a given generating volume if a rigid lid, as opposed to a free surface, was used. Mathematical models of ISWs routinely employ a rigid lid approximation - a condition regarded as being representative of the ocean where surface tension effects are negligible. The upper boundary condition clearly plays an important 
role and while the effect of surface tension in the ocean may be negligible that of surface waves is not. Exactly how surface waves effect the stability and propagation properties of ISWs in an oceanic context awaits investigation. Two oceanographic effects which may be of particular relevance in this context are (i) surface convergence ahead of the descending trough of an nonlinear internal wave in which surface gravity waves may become shorter and steeper during light to moderate wind conditions and (ii) surface capillary wave damping in the surface convergence, in windless conditions and in the presence of natural surfactants (biogenic oils).

Experimental observations were made of highly unstable ISWs. At the viewing location consequences of instability were often violent and well developed. Significant discrepancies were seen between the flow characteristics in these cases and those predicted theoretically for the onset of instability (see section IV). In particular, it was found that the unstable region of mixing grew in the horizontal extent and the horizontal velocity, $u / c$, diminished with wave instability. Such unexpected results highlight the need for a theoretical and experimental stability analysis of the fully nonlinear ISW equations. This paper has addressed the general stability characteristics of ISWs, a complimentary investigation of instability growth, development, and dissipation is now required.

\section{ACKNOWLEDGMENTS}

M. Carr and P. A. Davies are grateful for the support of a grant from the UK Engineering and Physical Sciences Research Council (EPSRC) under its Physics-Engineering Programme. Technical support at the University of Dundee was provided by John Anderson and Gary Conacher. The work of D.Fructus, J. Grue and A. Jensen was suppported by the Reseach Council of Norway, through the Strategic University Programme: Modelling of currents and waves for sea structures, at the University of Oslo. Technical support at the University of 
Oslo was provided by Svein Vesterby and Arve Kvalheim.

The authors would like to thank two anonymous referees for critical comments that have led to improvements in the paper.

\section{References}

[1] K. R. Helfrich and W. K. Melville, "Long nonlinear internal waves," Annu. Rev. Fluid. Mech. 38, 395 (2006).

[2] J. N. Moum, D. M. Farmer, W. D. Smyth, L. Armi and S. Vagle, "Structure and generation of turbulence at interfaces strained by internal solitary waves propagating shoreward over the continental shelf," J. Phys. Oceanogr. 33, 2093 (2003).

[3] J. R. Apel, J. R. Holbrook, A. K. Liu and J. Tsai, "The Sulu Sea internal soliton experiment," J. Phys. Oceanogr. 15, 1625 (1985).

[4] D. M. Farmer and J. D. Smith, "Tidal interaction of stratified flow with a sill in Knight Inlet," Deep-Sea Res. 27A, 239 (1980).

[5] J. Grue, A. Jensen, P. O. Rusȧs and J. K. Sveen, "Breaking and broadening of internal solitary waves," J. Fluid Mech. 413, 181 (2000).

[6] K. G. Lamb, "A numerical investigation of solitary internal waves with trapped cores formed via shoaling," J. Fluid Mech. 451, 109 (2000).

[7] K. G. Lamb and K. P. Wilkie, "Conjugate flows for waves with trapped cores," Phys. Fluids. 16(12), 4685 (2004).

[8] D. Fructus and J. Grue, "Fully nonlinear solitary waves in a layered stratified fluid," J. Fluid Mech. 505, 323 (2004). 
[9] D. Fructus, M. Carr, J. Grue, A. Jensen and P. A. Davies, "Shear-induced breaking of large internal solitary waves," J. Fluid Mech. In the press (2008).

[10] J. M. H. Fortuin, "Theory and application of two supplementary methods for constructing density gradient columns," J. Polym. Sci. 44, 505 (1960).

[11] P. A. Davies, "Aspects of flow visualisation and density field monitoring of stratified flows, Optics and Lasers in Engineering," 16, 311 (1992).

[12] J. Grue, A. Jensen, A., P. O. Rusȧs and J. K. Sveen, "Properties of large amplitude internal waves," J. Fluid Mech. 380, 257 (1999).

[13] T. W. Kao, F. S. Pan and D. Renouard, "Internal solitons on the pycnocline: generation, propagation, and shoaling and breaking over a slope," J. Fluid Mech. 159, 19 (1985).

[14] S. B. Dalziel, "DigiFlow Users' Guide," Advanced Image Processing for Fluid Mechanics, Dalziel Research Partners, 142 Cottenham Road, Histon, Cambridge CB24 9ET, England, available at www.dampt.cam.ac.uk/lab/digiflow/ as of May, 2008.

[15] J. W. Miles, "On stability of heterogeneous shear flows," J. Fluid Mech. 10, 496 (1961).

[16] L. N. Howard, "Note on a paper by John W. Miles," J. Fluid Mech. 10, 509 (1961).

[17] S. B. Dalziel, M. Carr, J. K. Sveen and P. A. Davies, "Simultaneous synthetic schlieren and PIV measurements for internal solitary waves," Meas. Sci. Technol. 18, 533 (2006).

[18] M. Stastna and K.G. Lamb, "Large fully nonlinear internal solitary waves: The effect of background current," Phys. Fluids. 14(9), 2987 (2002). 